TRANSACTIONS OF THE

AMERICAN MATHEMATICAL SOCIETY

Volume 356, Number 5 , Pages 1751-1779

S 0002-9947(03)03507-4

Article electronically published on December 15, 2003

\title{
NEWTON POLYHEDRA, UNSTABLE FACES AND THE POLES OF IGUSA'S LOCAL ZETA FUNCTION
}

\author{
KATHLEEN HOORNAERT
}

\begin{abstract}
In this paper we examine when the order of a pole of Igusa's local zeta function associated to a polynomial $f$ is smaller than "expected". We carry out this study in the case that $f$ is sufficiently non-degenerate with respect to its Newton polyhedron $\Gamma(f)$, and the main result of this paper is a proof of one of the conjectures of Denef and Sargos. Our technique consists in reducing our question about the polynomial $f$ to the same question about polynomials $f_{\mu}$, where $\mu$ are faces of $\Gamma(f)$ depending on the examined pole and $f_{\mu}$ is obtained from $f$ by throwing away all monomials of $f$ whose exponents do not belong to $\mu$. Secondly, we obtain a formula for Igusa's local zeta function associated to a polynomial $f_{\mu}$, with $\mu$ unstable, which shows that, in this case, the upperbound for the order of the examined pole is obviously smaller than "expected".
\end{abstract}

\section{INTRODUCTION}

For $p$ prime, denote the field of $p$-adic numbers by $\mathbb{Q}_{p}$, the ring of $p$-adic integers by $\mathbb{Z}_{p}$, and the finite field of $p$ elements by $\mathbb{F}_{p}$. If $R$ is a commutative ring with identity, we denote the set of its units by $R^{\times}$.

Definition 1.1. Let $f(x)=f\left(x_{1}, \ldots, x_{n}\right) \in \mathbb{Z}_{p}\left[x_{1}, \ldots, x_{n}\right]$ with $p$ prime. For $z \in \mathbb{Q}_{p}$, ord $z \in \mathbb{Z} \cup\{\infty\}$ denotes the valuation, $|z|=p^{- \text {ord } z}$ and ac $(z)=p^{- \text {ord } z} z$ denotes the angular component. Let $\chi: \mathbb{Z}_{p}^{\times} \rightarrow \mathbb{C}^{\times}$be a character of $\mathbb{Z}_{p}^{\times}$, i.e., a group homomorphism with finite image. We formally set $\chi(0)=0$. To the above data we associate the following two Igusa local zeta functions (the global and the local functions):

$$
\begin{aligned}
Z_{f}(s, \chi) & =\int_{\mathbb{Z}_{p}^{n}} \chi(\operatorname{ac} f(x))|f(x)|^{s}|d x|, \\
Z_{f, 0}(s, \chi) & =\int_{\left(p \mathbb{Z}_{p}\right)^{n}} \chi(\operatorname{ac} f(x))|f(x)|^{s}|d x|,
\end{aligned}
$$

for $s \in \mathbb{C}, \operatorname{Re}(s)>0$, where $|d x|$ denotes the Haar measure on $\mathbb{Q}_{p}^{n}$ normalized so that $\mathbb{Z}_{p}^{n}$ has measure 1 . If $\chi$ is the trivial character $\chi_{\text {triv }}$, then we write $Z_{f}(s)$ (resp. $\left.Z_{f, 0}(s)\right)$ instead of $Z_{f}\left(s, \chi_{\text {triv }}\right)\left(\right.$ resp. $\left.Z_{f, 0}\left(s, \chi_{\text {triv }}\right)\right)$.

Received by the editors March 12, 2002.

2000 Mathematics Subject Classification. Primary 11S40, 11D79; Secondary 14M25, 52B20, $14 \mathrm{G} 10$.

Key words and phrases. Igusa zeta function, Newton polyhedron, congruences, $p$-adic integrals. 
Igusa's local zeta function $Z_{f}(s)$ is directly related to the number of solutions of the congruences $f(x) \equiv 0 \bmod p^{m}, m=1,2,3, \ldots$; see, e.g., Igu78, pp. 9798], Den91, Section 1.2]. Using resolutions of singularities, Igusa Igu74 proved that $Z_{f}(s, \chi)$ is a rational function of $p^{-s}$ (see also Igu78]). An entirely different proof was obtained ten years later by Denef [Den84 using $p$-adic cell decomposition instead of resolutions of singularities. We denote the meromorphic continuation of $Z_{f}(s, \chi)$ again by $Z_{f}(s, \chi)$. We study the poles of Igusa's local zeta function for a special but important class of polynomials, namely the polynomials that are nondegenerate with respect to their Newton polyhedron. This study was first started by Lichtin and Meuser [LM85] for polynomials in two variables.

Definition 1.2. Let $f(x)=f\left(x_{1}, \ldots, x_{n}\right)=\sum_{\omega \in \mathbb{N}^{n}} a_{\omega} x_{1}^{\omega_{1}} \cdots x_{n}^{\omega_{n}}$ be a non-zero polynomial over $\mathbb{Z}_{p}$ with $f(0)=0$. Let $\mathbb{R}^{+}=\{x \in \mathbb{R} \mid x \geq 0\}$, and let $\operatorname{supp}(f)=$ $\left\{\omega \in \mathbb{N}^{n} \mid a_{\omega} \neq 0\right\}$ be the support of $f$. The Newton polyhedron $\Gamma(f)$ of $f$ is defined as the convex hull in $\left(\mathbb{R}^{+}\right)^{n}$ of the set

$$
\bigcup_{\omega \in \operatorname{supp}(f)} \omega+\left(\mathbb{R}^{+}\right)^{n}
$$

The global Newton polyhedron $\Gamma_{\mathrm{gl}}(f)$ of $f$ is defined as the convex hull of $\operatorname{supp}(f)$. It is easy to verify that $\Gamma(f)=\Gamma_{\mathrm{gl}}(f)+\left(\mathbb{R}^{+}\right)^{n}$.

Because a Newton polyhedron is a polyhedron, every proper face is an exposed face 1 So, every proper face of $\Gamma(f)$ is the intersection of $\Gamma(f)$ with a supporting hyperplane [Roc70, pp. 99-100]. By the faces of $\Gamma(\mathbf{f})$ we mean the proper faces of $\Gamma(f)$ and the Newton polyhedron $\Gamma(f)$ itself.

Definition 1.3. Let $p$ be a prime number. Let $f$ be as in Definition 1.2. For each face $\tau$ of the Newton polyhedron $\Gamma(f)$ of $f$, we define

$$
f_{\tau}(x)=\sum_{\omega \in \tau} a_{\omega} x^{\omega}
$$

and we also define the polynomial $\bar{f}_{\tau}(x)$ with coefficients in $\mathbb{F}_{p}$ by reducing each coefficient $a_{\omega}$ of $f_{\tau}$ modulo $p \mathbb{Z}_{p}$.

We say $f$ is non-degenerate over $\mathbb{F}_{p}$ with respect to all the faces of its Newton polyhedron $\Gamma(f)$ if for every face $\tau$ of $\Gamma(f)$ the locus of the polynomial $\bar{f}_{\tau}$ has no singularities in $\left(\mathbb{F}_{p}^{\times}\right)^{n}$, or equivalently the set of congruences

$$
\left\{\begin{array}{l}
f_{\tau}(x) \equiv 0 \bmod p \\
\frac{\partial f_{\tau}}{\partial x_{i}}(x) \equiv 0 \bmod p, \quad i=1, \ldots, n,
\end{array}\right.
$$

has no solution in $\left(\mathbb{Z}_{p}^{\times}\right)^{n}$.

We say $f$ is non-degenerate over $\mathbb{F}_{p}$ with respect to the compact faces of its Newton polyhedron if we have the same condition, but only for the compact faces of $\Gamma(f)$.

See [DH01] for some remarks about the definition of non-degenerate. In [DH01] and Hoo02] (see Theorem 3.2) we find an explicit formula for $Z_{f}(s, \chi)$ that holds if $f$ is non-degenerate over $\mathbb{F}_{p}$ with respect to all the faces of its Newton polyhedron and $\chi$ is a character of $\mathbb{Z}_{p}$ with conductor $c_{\chi}=1$. The conductor $c_{\chi}$ is the smallest $c \in \mathbb{N} \backslash\{0\}$ such that $\chi$ is trivial on $1+p^{c} \mathbb{Z}_{p}$. So, $c_{\chi \text { triv }}$ is also equal to 1 . It

\footnotetext{
${ }^{1}$ Face and exposed face in the sense of [Roc70 p. 162].

${ }^{2}$ Thus also for $\Gamma(f)$.
} 
is well known (see [Den91. Theorem 3.3]) that $Z_{f}(s, \chi)$ is constant as a function of $s$ for $p \gg 0$ if the conductor $c_{\chi}>1$ and $f$ is a polynomial over $\mathbb{Z}$. For the exact condition of a similar result for $f$ a polynomial over $\mathbb{Z}_{p}$ and $p$ a fixed prime number, we refer to the same reference. So, we have an explicit formula for $Z_{f}(s, \chi)$ for the relevant cases. The actors in the above-mentioned formula are the cones $\Delta_{\tau}$ associated to the faces $\tau$ of $\Gamma(f)$ (see Definition 2.3) and, the number of elements $N_{\tau}$ of the sets $\left\{x \in\left(\mathbb{F}_{p}^{\times}\right)^{n} \mid \bar{f}_{\tau}(x)=0\right\}$ if $\chi=\chi_{\text {triv }}$, or the sums $\sum_{a \in\left(\mathbb{F}_{p}^{\times}\right)^{n}} \chi\left(f_{\tau}(a)\right)$ if $\chi \neq \chi_{\text {triv }}$. Zúñiga-Galindo [Zn99] obtained the same formula, as a special case of a more general result. For the related work of other authors we refer to [DH01] and the references therein. Using the formula from Theorem 3.2, we can get a set of possible poles for $Z_{f}(s, \chi)$ (which we call candidate poles) together with their expected orders, i.e., upper bounds for the orders if these candidate poles are actual poles (see Proposition 4.1, Definition 4.2 Proposition4.5 and Proposition4.7). This can all be expressed in terms of the faces of $\Gamma(f)$ and their dimensions.

We have special interest for the largest real candidate pole of $Z_{f}(s, \chi)$ different from -1. It is well known (see Proposition 4.5) that this candidate pole equals $-1 / t_{0}$ if $t_{0} \neq 1$, and that its expected order equals $\kappa$, where $\left(t_{0}, t_{0}, \ldots, t_{0}\right)$ is the intersection point of the diagonal $D=\left\{(t, t, \ldots, t) \in \mathbb{R}^{n}\right\}$ with $\Gamma(f)$, and $\kappa$ is the codimension of the smallest face $\tau_{0}$ of $\Gamma(f)$ that contains $\left(t_{0}, t_{0}, \ldots, t_{0}\right)$. The main purpose of this article is to prove one of the conjectures of Denef and Sargos DS92. Section 6.2] for non-degenerate polynomials in the $p$-adic case:

$$
\begin{aligned}
& \text { if } \tau_{0} \text { is an unstable face of } \Gamma(f) \text { over } \mathbb{F}_{p} \text { with respect to } f \text {, and if } \\
& -1 / t_{0} \neq-1 \text { is a pole of } Z_{f}(s, \chi) \text {, then its order will be smaller } \\
& \text { than expected, i.e., }<\kappa \text {. }
\end{aligned}
$$

The notion "unstable" (see Definition 7.1) was first established by Denef and Sargos in [DS92]. The analogue of the above conjecture for the real case has been proved by Denef, Laeremans and Sargos [DLS97]. Inspired by the proof for the real case, we tried to reduce our question about the polynomial $f$ to the same question about the polynomial $f_{\tau_{0}}$. Namely, Theorem [5.3, our first main result, states that $-1 / t_{0}$ is an actual pole of $Z_{f}(s, \chi)$ of the expected order if and only if the same holds for $Z_{f_{\tau_{0}}}(s, \chi)$. In particular, if $-1 / t_{0}$ is not a pole of $Z_{f_{\tau_{0}}}(s, \chi)$ of the expected order, the same holds for $Z_{f}(s, \chi)$. Theorem 6.1 extends this result to arbitrary poles.

In Theorem 7.3, we will give a formula for $Z_{f}(s, \chi)$ that holds if $f=f_{\sigma}$, where $\sigma \subset \Gamma(f)$ is an unstable convex set over $\mathbb{F}_{p}$ with respect to $f$ (see Definition 7.1), and if $f$ is non-degenerate over $\mathbb{F}_{p}$ with respect to a particular set of faces of $\Gamma(f)$. The formula in this theorem, which is our second main result, is similar to the one in Theorem [3.2 but can provide a smaller set of candidate poles for $Z_{f}(s, \chi)$ or smaller upperbounds for their orders (see Example 8.1). Finally, Theorem 7.6 uses this formula together with Theorem 5.3 to obtain a proof of the above-mentioned conjecture of Denef and Sargos. Theorem[7.7 gives an analogous result for arbitrary poles.

I would like to thank Jan Denef for suggesting that I solve the above conjecture and for supervising this work. We also refer to the papers [PSS99] and [PS00] which treat similar questions in the archimedean setting.

\section{More about Newton polyhedra}

In Definition [2.3, we give a partition of $\left(\mathbb{R}^{+}\right)^{n}$ in sets that are closely related to the Newton polyhedron of a polynomial $f$ as in Definition [1.2 In order to define 
these sets $\Delta_{\tau}$ and to know more about them, we will present a selection of some well-known definitions and properties.

Definition 2.1. Let $f$ be as in Definition 1.2 For $a \in\left(\mathbb{R}^{+}\right)^{n}$, we define

$$
m(a)=\inf _{x \in \Gamma(f)}\{a \cdot x\}
$$

and we define the first meet locus of $a$ as

$$
F(a)=\{x \in \Gamma(f) \mid a \cdot x=m(a)\},
$$

where $a \cdot x$ denotes the scalar product of $a$ and $x$.

Property 2.2. Let $f$ be as in Definition 1.2 and $a \in\left(\mathbb{R}^{+}\right)^{n}$. Then $F(a)$, the first meet locus of $a$, is a face of $\Gamma(f)$. In particular, $F(0)=\Gamma(f)$ and $F(a)$ is a proper face of $\Gamma(f)$, if $a \neq 0$. Moreover, $F(a)$ is a compact face if and only if $a \in\left(\mathbb{R}^{+} \backslash\{0\}\right)^{n}$.

Definition 2.3. We define an equivalence relation on $\left(\mathbb{R}^{+}\right)^{n}$ by

$$
a \sim a^{\prime} \quad \text { if and only if } \quad F(a)=F\left(a^{\prime}\right) .
$$

If $\tau$ is a face of $\Gamma(f)$, we define the cone associated to $\tau$ as

$$
\Delta_{\tau}=\left\{a \in\left(\mathbb{R}^{+}\right)^{n} \mid F(a)=\tau\right\} .
$$

Note that $\Delta_{\Gamma(f)}=\{0\}$. We now study the other equivalence classes $\Delta_{\tau}$ and their closures $\bar{\Delta}_{\tau}$. It is well known that $\Delta_{\tau}$ is a relatively open subset of $\left(\mathbb{R}^{+}\right)^{n}$ and that $\bar{\Delta}_{\tau}$ is equal to $\left\{a \in\left(\mathbb{R}^{+}\right)^{n} \mid F(a) \supset \tau\right\}$.

Definition 2.4. A fan $\mathcal{F}$ is a finite set of rational polyhedral cones such that

(1) every (non-empty) face of a cone of $\mathcal{F}$ is contained in $\mathcal{F}$ and

(2) the intersection of two arbitrary cones $C_{i}$ and $C_{j}$ in $\mathcal{F}$ is a face of both $C_{i}$ and $C_{j}$.

Lemma 2.5. Let $f$ be as in Definition 1.2. The closures $\bar{\Delta}_{\tau}$ of the cones associated to the faces of $\Gamma(f)$ form a fan in $\left(\mathbb{R}^{+}\right)^{n}$. Moreover, we have the following. Let $\tau$ be a proper face of $\Gamma(f)$. Then the order-reversing map

$$
\{\text { faces of } \Gamma(f) \text { that contain } \tau\} \rightarrow\left\{\left(\text { non-empty) faces of } \bar{\Delta}_{\tau}\right\}: \sigma \mapsto \bar{\Delta}_{\sigma}\right.
$$

is one-to-one and onto.

Definition 2.6. A vector $a \in \mathbb{R}^{n}$ is called primitive if the components of $a$ are integers whose greatest common divisor is 1 .

Note that because a Newton polyhedron is a polyhedron, we can prove that every proper face $\tau$ of $\Gamma(f)$ is the (finite) intersection of the facet 3 of $\Gamma(f)$ that contain $\tau$. One can also prove that for every facet of $\Gamma(f)$ there exists a unique primitive vector in $\mathbb{N}^{n} \backslash\{0\}$ that is perpendicular to that facet.

Proposition 2.7. Let $f$ be as in Definition 1.2. Let $\tau$ be a proper face of $\Gamma(f)$ and let $\gamma_{1}, \ldots, \gamma_{r}$ be the facets of $\Gamma(f)$ that contain $\tau$. Let $a_{1}, \ldots, a_{r}$ be the unique primitive vectors in $\mathbb{N}^{n} \backslash\{0\}$ that are perpendicular to $\gamma_{1}, \ldots, \gamma_{r}$, respectively. Then

${ }^{3}$ Recall that a facet is an $(n-1)$-dimensional face. 
the cones $\bar{\Delta}_{\tau}$ and $\Delta_{\tau}$ are the following convex cones:

$$
\bar{\Delta}_{\tau}=\left\{\sum_{i=1}^{r} \lambda_{i} a_{i} \mid \lambda_{i} \in \mathbb{R}, \lambda_{i} \geq 0\right\} \quad \text { and } \quad \Delta_{\tau}=\left\{\sum_{i=1}^{r} \lambda_{i} a_{i} \mid \lambda_{i} \in \mathbb{R}, \lambda_{i}>0\right\} .
$$

Moreover, $\operatorname{dim} \Delta_{\tau}=\operatorname{dim} \bar{\Delta}_{\tau}=\operatorname{codim} \tau=n-\operatorname{dim} \tau$.

Definition 2.8. If $a_{1}, \ldots, a_{r} \in \mathbb{R}^{n} \backslash\{0\}$, we call cone $\left(a_{1}, \ldots, a_{r}\right)=\left\{\sum_{i=1}^{r} \lambda_{i} a_{i} \mid\right.$ $\left.\lambda_{i} \in \mathbb{R}, \lambda_{i}>0\right\}$ the cone strictly positively spanned by the vectors $a_{1}, \ldots, a_{r}$. Fix a cone $\Delta$. If there exist linearly independent vectors $a_{1}, \ldots, a_{r} \in \mathbb{R}^{n} \backslash\{0\}$ such that $\Delta=$ cone $\left(a_{1}, \ldots, a_{r}\right)$, then $\Delta$ is called a simplicial cone. If moreover one can choose $a_{1}, \ldots, a_{r} \in \mathbb{Z}^{n}$ (or in $\mathbb{Q}^{n}$ ), we say $\Delta$ is a rational simplicial cone.

Let $\tau$ be a proper face of $\Gamma(f)$ and let $a_{1}, \ldots, a_{r}$ be as in Proposition 2.7. Following from Proposition 2.7 and Lemma 2.9] one can partition the cone $\Delta_{\tau}$ associated to the face $\tau$ into a finite number of rational simplicial cones such that each $\Delta_{i}$ is spanned by vectors from the set $\left\{a_{1}, \ldots, a_{r}\right\}$.

Lemma 2.9. Let $\Delta$ be the cone strictly positively spanned by the vectors $a_{1}, \ldots$, $a_{r} \in\left(\mathbb{R}^{+}\right)^{n} \backslash\{0\}$. Then there exists a finite partition of $\Delta$ into cones $\Delta_{i}$, such that each $\Delta_{i}$ is strictly positively spanned by some vectors from the set $\left\{a_{1}, \ldots, a_{r}\right\}$ that are linearly independent over $\mathbb{R}$.

Proof. [Den95, Lemma 2].

Definition 2.10. Let $a_{1}, \ldots, a_{r}$ be vectors in $\mathbb{Z}^{n}$ that are linearly independent over $\mathbb{Q}$. We define the multiplicity of $a_{1}, \ldots, a_{r}$ as the index of the lattice $\mathbb{Z} a_{1}+\cdots+\mathbb{Z} a_{r}$ in the group of the points with integral coordinates of the vector space generated by $a_{1}, \ldots, a_{r}$.

Remark. It is easy to verify that the multiplicity of $a_{1}, \ldots, a_{r}$ equals the number of elements in the set

$$
\mathbb{Z}^{n} \cap\left\{\sum_{i=1}^{r} \lambda_{i} a_{i} \mid 0 \leq \lambda_{i}<1 \text { for } i=1, \ldots, r\right\},
$$

and also equals the greatest common divisor of the determinants of all $r \times r$-matrices obtained by omitting columns from the matrix with rows $a_{1}, \ldots, a_{r}$.

\section{An EXPLicit FORMUla FOR $Z_{f}(s, \chi)$}

In Theorem 3.2 we give a formula for $Z_{f}(s, \chi)$ that holds if $f$ is non-degenerate over $\mathbb{F}_{p}$ with respect to all the faces of its Newton polyhedron. In Section 7 we will give a similar formula for $Z_{f}(s, \chi)$ that holds if $f=f_{\sigma}$, where $\sigma \subset \Gamma(f)$ is an unstable convex set, and if $f$ is non-degenerate over $\mathbb{F}_{p}$ with respect to a smaller set of faces of $\Gamma(f)$.

Definition 3.1. For $k=\left(k_{1}, \ldots, k_{n}\right) \in \mathbb{R}^{n}$, we define

$$
\sigma(k)=\sum_{i=1}^{n} k_{i} .
$$

Theorem 3.2. Let $p$ be a prime number. Let $f$ be as in Definition 1.2. Suppose that $f$ is non-degenerate over the finite field $\mathbb{F}_{p}$ with respect to all the faces of its 
Newton polyhedron $\Gamma(f)$. Let $\chi$ be a character of $\mathbb{Z}_{p}^{\times}$with conductor $c_{\chi}=1$. For each face $\tau$ of $\Gamma(f)$ denote by $N_{\tau}$ the number of elements in the set

$$
\left\{a \in\left(\mathbb{F}_{p}^{\times}\right)^{n} \mid \bar{f}_{\tau}(a)=0\right\} .
$$

Let $s$ be a complex variable with $\operatorname{Re}(s)>0$. Then

$$
Z_{f}(s, \chi)=\sum_{\substack{\tau \text { face } \\ \text { of } \Gamma(f)}} L_{\tau} S_{\Delta_{\tau}},
$$

with

$$
L_{\tau}= \begin{cases}p^{-n}\left((p-1)^{n}-p N_{\tau} \frac{p^{s}-1}{p^{s+1}-1}\right) & \text { for } \chi=\chi_{\text {triv }}, \\ p^{-n} \sum_{a \in\left(\mathbb{F}_{p}^{\times}\right)^{n}} \chi\left(f_{\tau}(a)\right) & \text { for } \chi \neq \chi_{\text {triv }}\end{cases}
$$

and

$$
S_{\Delta_{\tau}}=\sum_{k \in \mathbb{N}^{n} \cap \Delta_{\tau}} p^{-\sigma(k)-m(k) s}
$$

for each face $\tau$ of $\Gamma(f)$ (including $\tau=\Gamma(f)$ ).

We have $S_{\Delta_{\Gamma(f)}}=1$ and the other $S_{\Delta_{\tau}}$ can be calculated as follows. Take a partition of the cone $\Delta_{\tau}$ associated to the proper face $\tau$ into rational simplicial cones $\Delta_{i}$. Then clearly $S_{\Delta_{\tau}}=\sum_{i} S_{\Delta_{i}}$, where the summation is over the rational simplicial cones $\Delta_{i}$ and

$$
S_{\Delta_{i}}=\sum_{k \in \mathbb{N}^{n} \cap \Delta_{i}} p^{-\sigma(k)-m(k) s} .
$$

Let $\Delta_{i}$ be the cone strictly positively spanned by the linearly independent vectors $a_{1}, \ldots, a_{r} \in \mathbb{N}^{n} \backslash\{0\}$. Then

$$
S_{\Delta_{i}}=\frac{\sum_{h} p^{\sigma(h)+m(h) s}}{\left(p^{\sigma\left(a_{1}\right)+m\left(a_{1}\right) s}-1\right) \cdots\left(p^{\sigma\left(a_{r}\right)+m\left(a_{r}\right) s}-1\right)},
$$

where $h$ runs through the elements of the set

$$
\mathbb{Z}^{n} \cap\left\{\sum_{j=1}^{r} \lambda_{j} a_{j} \mid 0 \leq \lambda_{j}<1 \text { for } j=1, \ldots, r\right\} .
$$

In the theorem above, $m(\cdot)$ and $\sigma(\cdot)$ are defined as in Definitions 2.1 and 3.1 Remarks.

(1) Note that $S_{\Delta_{\tau}}$ is a rational function in $p^{-s}$. We also denote its meromorphic continuation by $S_{\Delta_{\tau}}$.

(2) We have

$$
Z_{f, 0}(s, \chi)=\sum_{\substack{\tau \text { compact face } \\ \text { of } \Gamma(f)}} L_{\tau} S_{\Delta_{\tau}},
$$

with $L_{\tau}$ and $S_{\Delta_{\tau}}$ as in the statement of the theorem above. For this formula, we only need $f$ to be non-degenerate over the finite field $\mathbb{F}_{p}$ with respect to the compact faces of $\Gamma(f)$.

Proof. [DH01, Theorem 4.2] and [Hoo02, Theorem 3.4]. 


\section{The CANDidate Poles of $Z_{f}(s, \chi)$ AND THEIR EXPECTED ORDERS}

Proposition 4.1. Suppose that the prime number $p$, the polynomial $f$ and the character $\chi$ satisfy the conditions of Theorem [3.2. Let $\gamma_{1}, \ldots, \gamma_{r}$ be all the facets of $\Gamma(f)$, and let $a_{1}, \ldots, a_{r}$ be the unique primitive vectors in $\mathbb{N}^{n} \backslash\{0\}$ that are perpendicular to $\gamma_{1}, \ldots, \gamma_{r}$, respectively. Then the following hold:

(i) If $s_{1}$ is a pole of $Z_{f}(s)$, then

$$
\begin{aligned}
& s_{1}=-1+i \frac{k 2 \pi}{\log _{e} p} \quad \text { with } k \in \mathbb{Z}, \text { or } \\
& s_{1}=-\frac{\sigma\left(a_{j}\right)}{m\left(a_{j}\right)}+i \frac{k 2 \pi}{m\left(a_{j}\right) \log _{e} p},
\end{aligned}
$$

with $k \in \mathbb{Z}$ and $j \in\{1, \ldots, r\}$ such that $m\left(a_{j}\right) \neq 0$.

(ii) If $s_{1}$ is a pole of $Z_{f}(s)$, then $\operatorname{Re}\left(s_{1}\right)$ is -1 or $\operatorname{Re}\left(s_{1}\right)$ is of the form $-1 / t_{1}$, where $\left(t_{1}, t_{1}, \ldots, t_{1}\right)$ is the intersection point of the diagonal $D=$ $\left\{(t, t, \ldots, t) \in \mathbb{R}^{n}\right\}$ with the supporting hyperplane of a facet of $\Gamma(f)$.

(iii) Suppose that $s_{1}$ is a pole of $Z_{f}(s, \chi)$ with $\chi \neq \chi_{\text {triv }}$. Then

$$
s_{1}=-\frac{\sigma\left(a_{j}\right)}{m\left(a_{j}\right)}+i \frac{k 2 \pi}{m\left(a_{j}\right) \log _{e} p},
$$

with $k \in \mathbb{Z}$ and $j \in\{1, \ldots, r\}$ such that $m\left(a_{j}\right) \neq 0$. Moreover, $\operatorname{Re}\left(s_{1}\right)$ is of the form $-1 / t_{1}$, where $\left(t_{1}, t_{1}, \ldots, t_{1}\right)$ is the intersection point of the diagonal $D=\left\{(t, t, \ldots, t) \in \mathbb{R}^{n}\right\}$ with the supporting hyperplane of a facet of $\Gamma(f)$.

Remarks.

(1) The same is true for $Z_{f, 0}(s)$ and $Z_{f, 0}(s, \chi)$. Of course, then we only need $f$ to be non-degenerate over $\mathbb{F}_{p}$ with respect to all the compact faces of $\Gamma(f)$ (see Remark (2) after Theorem 3.2).

(2) We call the complex numbers in Proposition 4.1(i) and (iii) the candidate poles of $Z_{f}(s)$ and $Z_{f}(s, \chi)$, respectively. The first candidate poles in (i) (with real part equal to -1 ) come from the $L_{\tau}$, the second ones in (i) and the ones in (iii) come from the $S_{\Delta_{\tau}}$. In the next section, we will give more information about the largest real candidate pole coming from the $S_{\Delta_{\tau}}$.

Proof. [DH01, Proposition 5.1].

Looking at the formula from Theorem 3.2, we can give upperbounds for the orders of the poles of $Z_{f}(s, \chi)$.

Definition 4.2. Suppose that the prime number $p$, the polynomial $f$ and the character $\chi$ satisfy the conditions of Theorem 3.2. Suppose that $s_{1}$ is a candidate pole of $Z_{f}(s, \chi)$, i.e., a number from the set described in Proposition 4.1. We define the expected order of the candidate pole $s_{1}$ for $Z_{f}(s, \chi)$ (according to the formula for $Z_{f}(s, \chi)$ in Theorem [3.2) as

$$
\operatorname{Max}\left\{\text { order of the pole } s_{1} \text { for } L_{\tau} S_{\Delta_{\tau}} \mid \tau \text { a face of } \Gamma(f)\right\},
$$

where the order of the pole $s_{1}$ is defined as 0 , if it is not a pole, and where $L_{\tau}$ and $S_{\Delta_{\tau}}$ are defined as in Theorem 3.2 If $\operatorname{Re}\left(s_{1}\right) \neq-1$ or $\chi \neq \chi_{\text {triv }}$, we omit $L_{\tau}$ in (4.1). 


\subsection{The largest real candidate pole coming from the $\boldsymbol{S}_{\boldsymbol{\Delta}_{\tau}}$.}

Definition 4.3. Let $f\left(x_{1}, \ldots, x_{n}\right)$ be a non-zero polynomial with $f(0)=0$. We denote the unique intersection point of the diagonal $D=\left\{(t, t, \ldots, t) \in \mathbb{R}^{n}\right\}$ with the boundary of the Newton polyhedron $\Gamma(f)$ of $f$ by $\left(t_{0}, t_{0}, \ldots, t_{0}\right)$. Denote the smallest face of $\Gamma(f)$ that contains $\left(t_{0}, t_{0}, \ldots, t_{0}\right)$ by $\tau_{0}$ and its codimension in $\mathbb{R}^{n}$ by $\kappa$. Put $\rho=-1 / t_{0}$.

We search for more information about the largest real candidate pole of $Z_{f}(s)$ and $Z_{f}(s, \chi)$ coming from the $S_{\Delta_{\tau}}$. Lemma 4.4 implies that this is $\rho$ and Proposition 4.5 shows that the expected order of $\rho$ for $Z_{f}(s)$ and $Z_{f}(s, \chi)$ is either $\kappa$ or $\kappa+1$.

Lemma 4.4. Let $f(x)=f\left(x_{1}, \ldots, x_{n}\right)$ be a non-zero polynomial with $f(0)=0$. For every $a \in\left(\mathbb{R}^{+}\right)^{n}$ we have $\sigma(a)-m(a)\left(1 / t_{0}\right) \geq 0$ with equality if and only if $\tau_{0} \subset F(a)$ (or equivalently $a \in \bar{\Delta}_{\tau_{0}}$ ).

Proof. See [DH01, Lemma 5.3].

Proposition 4.5. Suppose that the prime number $p$, the polynomial $f$ and the character $\chi$ satisfy the conditions of Theorem 3.2. Then for every pole $s_{1}$ of $Z_{f}(s, \chi)$, one has $\operatorname{Re}\left(s_{1}\right) \leq \rho$ if $\chi \neq \chi_{\text {triv }}$; otherwise $\operatorname{Re}\left(s_{1}\right)=-1$ or $\operatorname{Re}\left(s_{1}\right) \leq \rho$. Moreover, the expected order of the candidate pole $\rho$ for $Z_{f}(s, \chi)$ equals $\kappa+1$ if $\chi=\chi_{\text {triv }}$, $\rho=-1$ and there is a face $\tau \subset \tau_{0}$ such that $N_{\tau} \neq 0$; otherwise, its expected order will be $\kappa$.

Recall that the actual order is not larger than the expected order.

Proof. See [DH01, Proposition 5.5] or [Zn99]. The case of the trivial character is also a direct consequence of the material in [Den95].

\subsection{The expected order of an arbitrary pole of $Z_{f}(s, \chi)$.}

Definition 4.6. Suppose that the prime number $p$, the polynomial $f$ and the character $\chi$ satisfy the conditions of Theorem 3.2. Suppose that $s_{1}$ is a candidate pole of $Z_{f}(s, \chi)$, i.e., a number from the set described in Proposition 4.1 We say that a vector $a \in\left(\mathbb{R}^{+}\right)^{n} \backslash\{0\}$ or a facet $F(a)$ contributes to the candidate pole $s_{1}$ if $p^{\sigma(a)+m(a) s_{1}}=1$, or equivalently, $\operatorname{Re}\left(s_{1}\right)=-\sigma(a) / m(a)$ and there exists a $k \in \mathbb{Z}$ such that $\operatorname{Im}\left(s_{1}\right)=k 2 \pi /\left(m(a) \log _{e} p\right)$. We say that a face of $\Gamma(f)$ is a face of pure contribution to the candidate pole $s_{1}$ if every facet that contains $\tau$ contributes to this candidate pole $s_{1}$. We define $\kappa\left(s_{1}\right)$ as the maximum of the codimensions of such faces. We call such a face of codimension $\kappa\left(s_{1}\right)$ a face of $\Gamma(f)$ of maximal pure contribution to the candidate pole.

Remarks.

(1) It can occur that not all faces of $\Gamma(f)$ of maximal pure contribution to the candidate pole $s_{1}$ are disjoint. See Example 8.2.

(2) If $s_{1}=\rho$, then $\kappa\left(s_{1}\right)=\kappa$ and $\tau_{0}$ is the only face of $\Gamma(f)$ of maximal pure contribution to the candidate pole $\rho$ of $Z_{f}(s, \chi)$.

Proposition 4.7. Suppose that the prime number $p$, the polynomial $f$ and the character $\chi$ satisfy the conditions of Theorem [3.2. Suppose that $s_{1}$ is a candidate pole of $Z_{f}(s, \chi)$, i.e., a number from the set described in Proposition 4.1. 
(i) Let $\mu$ be a face of $\Gamma(f)$ of pure contribution to the candidate pole $s_{1}$ and suppose that its codimension is $k$. There exists a constant $c_{\mu}>0$, independent of $p$, such that

$$
\lim _{s \rightarrow s_{1}}\left(p^{s-s_{1}}-1\right)^{k} S_{\Delta_{\mu}}=c_{\mu} .
$$

Define the half-space $H_{1}^{-}=\left\{x \in \mathbb{R}^{n} \mid \sum_{i=1}^{n} x_{i} \leq 1\right\}$ and define $\omega_{\bar{\Delta}_{\mu}}$ as the volume form on the vector space vct $\bar{\Delta}_{\mu}$ generated by $\bar{\Delta}_{\mu}$, normalized such that the parallelopiped spanned by a lattice basis of $\mathbb{Z}^{n} \cap \operatorname{vct} \bar{\Delta}_{\mu}$ has volume 1. Then

$$
c_{\mu}=k !\left|\operatorname{Re}\left(s_{1}\right)\right|^{k} \operatorname{Vol}_{\omega_{\bar{\Delta}_{\mu}}}\left(\bar{\Delta}_{\mu} \cap H_{1}^{-}\right),
$$

where the volume is taken with respect to $\omega_{\bar{\Delta}_{\mu}}$.

(ii) If $p^{s_{1}+1} \neq 1$ or $\chi \neq \chi_{\text {triv }}$, then the expected order of the candidate pole $s_{1}$ of $Z_{f}(s, \chi)$ is $\kappa\left(s_{1}\right)$. Otherwise, the expected order is either $\kappa\left(s_{1}\right)$ or $\kappa\left(s_{1}\right)+1$.

Proof. [Hoo02, Lemma 4.8 and Proposition 4.9].

\section{Checking if $\rho=-1 / t_{0}$ IS A POle OF THE EXPECTED ORDeR: REDUCTION FROM $f$ TO $f_{\tau_{0}}$}

Definition 5.1. Let $V=\left\{v_{1}, \ldots, v_{r}\right\}$ be a finite subset of $\mathbb{Z}^{n}$ such that its elements are linearly independent over $\mathbb{Q}$. Define

$$
H_{V}=H_{\left\{v_{1}, \ldots, v_{r}\right\}}=\mathbb{Z}^{n} \cap\left\{\sum_{i=1}^{r} \lambda_{i} v_{i} \mid 0 \leq \lambda_{i}<1 \text { for } i=1, \ldots, r\right\} .
$$

Note that all $\lambda_{i}$ belong to $\mathbb{Q}$.

Recall that we can choose a unique representative in $H_{\left\{v_{1}, \ldots, v_{r}\right\}}$ for a coset of the lattice $\mathbb{Z} v_{1}+\cdots+\mathbb{Z} v_{r}$ in the group of the points with integral coordinates of the vector space generated by $v_{1}, \ldots, v_{r}$. So, the number of elements in $H_{\left\{v_{1}, \ldots, v_{r}\right\}}$ equals the multiplicity of $v_{1}, \ldots, v_{r}$.

In the formula for Igusa's local zeta function from Theorem 3.2, we have to calculate sums of the form $\sum_{h} p^{\sigma(h)+m(h) s}$, where $h$ runs through the elements of a set of the form $H_{\left\{v_{1}, \ldots, v_{r}\right\}}$. The next lemma gives a relation between those sets if some of the vectors from $\left\{v_{1}, \ldots, v_{r}\right\}$ are the same and the others generate the same vector space. We use this relation in the proof of Theorem 5.3, which shows that $\rho \neq-1$ is a pole of $Z_{f}(s, \chi)$ of the expected order (see Proposition 4.5) if and only if the same holds for $Z_{f_{\tau_{0}}}(s, \chi)$. This is very surprising because the cones $\Delta_{\tau}$, and hence the $S_{\Delta_{\tau}}$ for $\tau \subset \tau_{0}$ in the formulas for $Z_{f}(s, \chi)$ and $Z_{f_{\tau_{0}}}(s, \chi)$ from Theorem 3.2 can be different. For example, let $f(x, y, z)=x y+x z^{2}+y z^{2}+z^{5}+y^{7}$. Then $\tau_{0}$ is the convex hull of $(1,1,0),(1,0,2)$ and $(0,1,2)$. So, $f_{\tau_{0}}=x y+x z^{2}+y z^{2}$ and

$$
\begin{aligned}
\Delta_{(0,1,2)}(f) & =\text { cone }((1,0,0),(2,2,1),(3,3,1),(6,1,3)), \\
\Delta_{(0,1,2)}\left(f_{\tau_{0}}\right) & =\text { cone }((1,0,0),(2,2,1),(1,1,0),(2,0,1)) .
\end{aligned}
$$

See also Figure 5.1 and Figure 8.2 . In the case that the codimension of $\tau_{0}$ is 1 , one can easily prove Theorem 5.3 . The real difficulties only occur when its codimension is larger than 1 . 


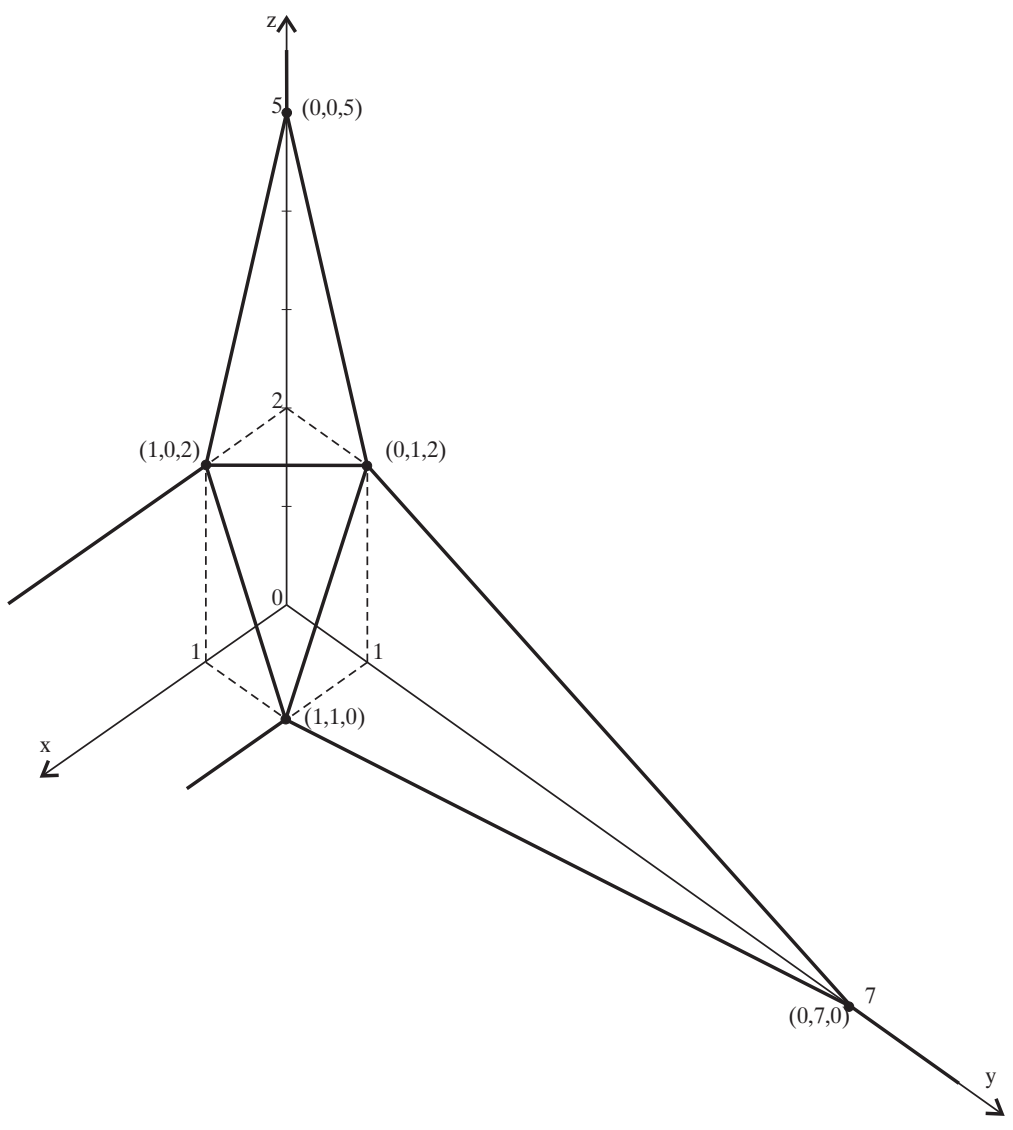

FiguRE 5.1. Newton polyhedron of $x y+x z^{2}+y z^{2}+z^{5}+y^{7}$

Lemma 5.2. Let $A, A^{\prime}$ and $B$ be finite subsets of $\mathbb{Z}^{n}$ such that each of the three sets $A, A^{\prime}$ and $A \cup B$ consists of linearly independent elements over $\mathbb{Q}$ and such that $A$ and $A^{\prime}$ generate the same vector space. Let $\pi$ be the natural projection of $\operatorname{vct} A+\operatorname{vct} B$ onto vct $B$ (here "vct" denotes "the vector space generated by"). Then we have

(i) $\#\left(\pi^{-1}(\{b\}) \cap H_{A \cup B}\right)=\# H_{A}$ for any $b \in \pi\left(H_{A \cup B}\right)$, and

(ii) $\pi\left(H_{A \cup B}\right)=\pi\left(H_{A^{\prime} \cup B}\right)$.

Proof. Let $A=\left\{a_{1}, \ldots, a_{u}\right\}, A^{\prime}=\left\{a_{1}^{\prime}, \ldots, a_{u}^{\prime}\right\}$ and $B=\left\{b_{1}, \ldots, b_{v}\right\}$.

(i) Suppose that $h=\sum_{i=1}^{u} \alpha_{i} a_{i}+b \in H_{A \cup B}$ with $b=\sum_{j=1}^{v} \beta_{j} b_{j}$. Suppose that $g=\sum_{i=1}^{u} \gamma_{i} a_{i}+b \in\left(\pi^{-1}(\{b\}) \cap H_{A \cup B}\right)$. Then $g-h \in \mathbb{Z}^{n} \cap \operatorname{vct}\left\{a_{1}, \ldots, a_{u}\right\}$. So, there exists a unique $k \in H_{A}$ and unique elements $z_{1}, \ldots, z_{u} \in \mathbb{Z}$ such that $g=h+k+\sum_{i=1}^{u} z_{i} a_{i}$ because $a_{1}, \ldots, a_{u} \in \mathbb{Z}^{n}$ are linearly independent. Conversely, if $k \in H_{A}$, there exists a unique $g \in\left(\pi^{-1}(\{b\}) \cap H_{A \cup B}\right)$ and unique elements $z_{1}, \ldots, z_{u} \in \mathbb{Z}$ such that $g=h+k+\sum_{i=1}^{u} z_{i} a_{i}$. This shows that $\#\left(\pi^{-1}(\{b\}) \cap H_{A \cup B}\right)=\# H_{A}$.

(ii) By the symmetry of the problem, it suffices to prove that $\pi\left(H_{A \cup B}\right) \subset$ $\pi\left(H_{A^{\prime} \cup B}\right)$. Suppose that $h=\sum_{i=1}^{u} \alpha_{i} a_{i}+b \in H_{A \cup B}$ with $b=\sum_{j=1}^{v} \beta_{j} b_{j}$. 
We now show that there also exists an element $h^{\prime}=\sum_{i=1}^{u} \alpha_{i}^{\prime} a_{i}^{\prime}+b \in H_{A^{\prime} \cup B}$, such that $\pi\left(h^{\prime}\right)=b=\pi(h)$. Because $a_{1}^{\prime}, \ldots, a_{u}^{\prime}$ generate the same vector space over $\mathbb{Q}$ as $a_{1}, \ldots, a_{u}$, there exist $\gamma_{1}, \ldots, \gamma_{u} \in \mathbb{Q}$ such that $\sum_{i=1}^{u} \alpha_{i} a_{i}=$ $\sum_{i=1}^{u} \gamma_{i} a_{i}^{\prime}$. For each $i$, there exists a unique $0 \leq \alpha_{i}^{\prime}<1$ and a $z_{i} \in \mathbb{Z}$ such that $\gamma_{i}=\alpha_{i}^{\prime}+z_{i}$. Because the elements $a_{1}^{\prime}, \ldots, a_{u}^{\prime}$ belong to $\mathbb{Z}^{n}$, it is clear that $h^{\prime}=\sum_{i=1}^{u} \alpha_{i}^{\prime} a_{i}^{\prime}+b=h-\sum_{i=1}^{u} z_{i} a_{i}^{\prime}$ is an element of $\mathbb{Z}^{n}$ and hence, also of $H_{A^{\prime} \cup B}$.

Theorem 5.3. Suppose that the prime number $p$, the polynomial $f$ and the character $\chi$ satisfy the conditions of Theorem [3.2. Let $\tau_{0}, \rho$ and $\kappa$ be defined as in Definition 4.3. Then

$$
\lim _{s \rightarrow \rho}\left(p^{s-\rho}-1\right)^{k} Z_{f}(s, \chi)=c \lim _{s \rightarrow \rho}\left(p^{s-\rho}-1\right)^{k} Z_{f_{\tau_{0}}}(s, \chi),
$$

where

$$
k= \begin{cases}\kappa+1 & \text { if } \rho=-1 \text { and } \chi=\chi_{\text {triv }} \\ \kappa & \text { otherwise }\end{cases}
$$

and $c>0$ is a constant real number, independent of $p$. In fact,

$$
c=\frac{\operatorname{Vol}\left(\bar{\Delta}_{\tau_{0}}(f) \cap H_{1}^{-}\right)}{\operatorname{Vol}\left(\bar{\Delta}_{\tau_{0}}\left(f_{\tau_{0}}\right) \cap H_{1}^{-}\right)},
$$

where the volume is the one on the vector space generated by $\bar{\Delta}_{\tau_{0}}$, induced by the standard metric on $\mathbb{R}^{n}$, and $H_{1}^{-}$is the half-space $\left\{x \in \mathbb{R}^{n} \mid \sum_{i=1}^{n} x_{i} \leq 1\right\}$.

Remarks.

(1) If it is important to know whether we examine the cone $\Delta_{\tau}$ associated to the polynomial $f$ or to the polynomial $f_{\tau_{0}}$, we will indicate this by $\Delta_{\tau}(f)$ and $\Delta_{\tau}\left(f_{\tau_{0}}\right)$, respectively.

(2) The proof below also shows that a similar result holds for $Z_{f, 0}(s, \chi)$, even if $\tau_{0}$ is not compact. Then $f$ has to be non-degenerate over $\mathbb{F}_{p}$ with respect to all the compact faces of its Newton polyhedron.

(3) If $\chi=\chi_{\text {triv }}, \rho=-1$ and the expected order of the candidate pole $\rho$ for $Z_{f}(s)$ equals $\kappa$, we know by Hoo02, Theorem 4.10] that $\rho$ is an actual pole of $Z_{f}(s)$ of order $\kappa$. So, Theorem 5.3 will be useful for the other cases.

Proof. Note also that $f_{\tau_{0}}$ is non-degenerate over the finite field $\mathbb{F}_{p}$ with respect to all the faces of its Newton polyhedron $\Gamma\left(f_{\tau_{0}}\right)$. Indeed, suppose that $\tau$ is a face of $\Gamma\left(f_{\tau_{0}}\right)$. Then $\left(f_{\tau_{0}}\right)_{\tau}=f_{\tau_{0} \cap \tau}$ and the face $\tau_{0} \cap \tau$ of $\tau_{0}$ is also a face of the Newton polyhedron $\Gamma(f)$, by [Roc70, p. 165]. So, we can use the formula for $Z_{f}(s, \chi)$ and $Z_{f_{\tau_{0}}}(s, \chi)$ from Theorem 3.2.

We know from [DH01, Lemma 5.4] that $\lim _{s \rightarrow \rho}\left(p^{s-\rho}-1\right)^{\kappa} S_{\Delta_{\tau}}=0$ for faces $\tau \not \subset$ $\tau_{0}$ and that $\lim _{s \rightarrow \rho}\left(p^{s-\rho}-1\right)^{\kappa} S_{\Delta_{\tau}}>0$ for faces $\tau \subset \tau_{0}$. Moreover, $L_{\tau}(f)=L_{\tau}\left(f_{\tau_{0}}\right)$ for faces $\tau \subset \tau_{0}$. So, it suffices to prove that

$$
\frac{\lim _{s \rightarrow \rho}\left(p^{s-\rho}-1\right)^{\kappa} S_{\Delta_{\tau}(f)}}{\lim _{s \rightarrow \rho}\left(p^{s-\rho}-1\right)^{\kappa} S_{\Delta_{\tau_{0}}(f)}}=\frac{\lim _{s \rightarrow \rho}\left(p^{s-\rho}-1\right)^{\kappa} S_{\Delta_{\tau}\left(f_{\tau_{0}}\right)}}{\lim _{s \rightarrow \rho}\left(p^{s-\rho}-1\right)^{\kappa} S_{\Delta_{\tau_{0}}\left(f_{\tau_{0}}\right)}}
$$

for faces $\tau \subset \tau_{0}$, and that

$$
\frac{\lim _{s \rightarrow \rho}\left(p^{s-\rho}-1\right)^{\kappa} S_{\Delta_{\tau_{0}}(f)}}{\lim _{s \rightarrow \rho}\left(p^{s-\rho}-1\right)^{\kappa} S_{\Delta_{\tau_{0}}\left(f_{\tau_{0}}\right)}}=\frac{\operatorname{Vol}\left(\bar{\Delta}_{\tau_{0}}(f) \cap H_{1}^{-}\right)}{\operatorname{Vol}\left(\bar{\Delta}_{\tau_{0}}\left(f_{\tau_{0}}\right) \cap H_{1}^{-}\right)} .
$$


The last equality follows by Proposition 4.7 because $\lim _{s \rightarrow \rho}\left(p^{s-\rho}-1\right)^{\kappa} S_{\Delta_{\tau_{0}}}$ equals $\kappa !|\rho|^{\kappa}$ times the volume of $\bar{\Delta}_{\tau_{0}} \cap H_{1}^{-}$with respect to the volume form $\omega_{\bar{\Delta}_{\tau_{0}}}$, where $\omega_{\bar{\Delta}_{\tau_{0}}}$ is the volume form on vct $\bar{\Delta}_{\tau_{0}}$, normalized such that the parallelopiped spanned by a lattice basis of $\mathbb{Z}^{n} \cap$ vct $\bar{\Delta}_{\tau_{0}}$ has volume 1 . Because $\bar{\Delta}_{\tau_{0}}(f)$ and $\bar{\Delta}_{\tau_{0}}\left(f_{\tau_{0}}\right)$ generate the same vector space, it is clear that we can also take the quotient of the volumes induced by the standard metric on $\mathbb{R}^{n}$.

Suppose that $\bigcup_{i} \Delta_{i}$ is a finite partition of $\Delta_{\tau}$ into rational simplicial cones. Then $S_{\Delta_{\tau}}=\sum_{i} S_{\Delta_{i}}$. From the formula for $S_{\Delta_{i}}$ in Theorem 3.2, Lemma 4.4 and the fact that $\operatorname{dim} \bar{\Delta}_{\tau_{0}}=\kappa$, it follows that $\lim _{s \rightarrow \rho}\left(p^{s-\rho}-1\right)^{\kappa} S_{\Delta_{i}}>0$ if and only if (exactly) $\kappa$ generators of $\Delta_{i}$ belong to $\bar{\Delta}_{\tau_{0}}$; otherwise, this limit is zero. So, to prove equality (5.2), it is only important to know which cones in the partition have $\kappa$ generators in $\bar{\Delta}_{\tau_{0}}$. We will choose partitions of $\Delta_{\tau}(f)$ and $\Delta_{\tau}\left(f_{\tau_{0}}\right)$ that are closely related to some chosen partitions of $\Delta_{\tau_{0}}(f)$ and $\Delta_{\tau_{0}}\left(f_{\tau_{0}}\right)$, respectively.

Fix a finite partition of $\Delta_{\tau_{0}}(f)$ into rational simplicial cones and let

$$
\text { cone } A_{i} \text {, for } i=1, \ldots, \ell \text {, }
$$

be the only cones of dimension $\kappa$ (i.e., with $\kappa$ generators in $\left.\bar{\Delta}_{\tau_{0}}(f)\right)$ in this partition. Here, each $A_{i}$ is a set of $\kappa$ vectors in $\mathbb{N}^{n}$ that are linearly independent over $\mathbb{Q}$. Similarly, fix a finite partition of $\Delta_{\tau_{0}}\left(f_{\tau_{0}}\right)$ into rational simplicial cones, let

$$
\text { cone } A_{j}^{\prime} \text {, for } j=1, \ldots, m \text {, }
$$

be the only cones of dimension $\kappa$ in this partition, and let each $A_{j}^{\prime}$ be a set of $\kappa$ vectors in $\mathbb{N}^{n}$ that are linearly independent over $\mathbb{Q}$. Note that the sets $A_{i}$ and the sets $A_{j}^{\prime}$ all generate the same vector space.

It is clear that $\Delta_{\tau}(f) \subset \Delta_{\tau}\left(f_{\tau_{0}}\right)$ for every face $\tau \subset \tau_{0}$. Indeed, if $H_{a}=\{x \in$ $\left.\mathbb{R}^{n} \mid a \cdot x=b\right\}$ is a supporting hyperplane of $\Gamma(f)$ such that $H_{a} \cap \Gamma(f)=\tau$, then the same property holds if we replace $\Gamma(f)$ by $\Gamma\left(f_{\tau_{0}}\right)$. Fix now for each face $\tau \varsubsetneqq \tau_{0}$ a primitive vector

$$
b_{\tau} \in \Delta_{\tau}(f) \subset \Delta_{\tau}\left(f_{\tau_{0}}\right) .
$$

From Lemma 2.5] and Lemma [5.5 below, it follows by induction on $d$ that the following assertion is true for any $d$ in $\mathbb{N} \backslash\{0\}$.

Assertion 5.4. Suppose that the face $\tau \varsubsetneqq \tau_{0}$ and that $\operatorname{dim} \tau=\operatorname{dim} \tau_{0}-d$. Then there exists a partition of $\Delta_{\tau}(f)$ into rational simplicial cones, such that the only cones in this partition that have $\kappa$ generators in $\bar{\Delta}_{\tau_{0}}(f)$ are precisely the cones of the form

$$
\text { cone }\left(A_{i} \cup B_{\tau}\right) \text {, }
$$

where $i \in\{1, \ldots, \ell\}$ and $B_{\tau}$ is a set of the form $\left\{b_{\tau_{1}}, \ldots, b_{\tau_{v-1}}, b_{\tau}\right\}$, where $v \leq d$ and $v=d$ for the cones in this partition of dimension equal to $\operatorname{dim} \Delta_{\tau}(f), \tau \varsubsetneqq \tau_{v-1} \varsubsetneqq$ $\ldots \mp \tau_{1} \varsubsetneqq \tau_{0}$ are faces of $\tau_{0}$ and $b_{\tau_{1}}, \ldots, b_{\tau_{v-1}}, b_{\tau}$ are defined in (5.4). Similarly, there exists a partition of $\Delta_{\tau}\left(f_{\tau_{0}}\right)$ into rational simplicial cones such that the only cones in this partition that have $\kappa$ generators in $\bar{\Delta}_{\tau_{0}}\left(f_{\tau_{0}}\right)$ are precisely the cones of the form

$$
\text { cone }\left(A_{j}^{\prime} \cup B_{\tau}\right) \text {, }
$$

where $j \in\{1, \ldots, m\}$ and $B_{\tau}$ is defined as above.

Note that one adds the same sets $B_{\tau}$ to the sets $A_{j}^{\prime}$ as one adds to the sets $A_{i}$. Also note that $\tau \varsubsetneqq \tau_{v-1} \varsubsetneqq \cdots \varsubsetneqq \tau_{1} \varsubsetneqq \tau_{0}$ for faces of $\tau_{0}$ is equivalent to $\bar{\Delta}_{\tau} \nsupseteq$ 
$\bar{\Delta}_{\tau_{v-1}} \supseteq \cdots \supseteq \bar{\Delta}_{\tau_{1}} \supseteq \bar{\Delta}_{\tau_{0}}$ for faces of $\bar{\Delta}_{\tau}$ (Lemma [2.5), and that $\operatorname{dim} \tau=\operatorname{dim} \tau_{0}-d$ is equivalent to $\operatorname{dim} \bar{\Delta}_{\tau}=\operatorname{dim} \bar{\Delta}_{\tau_{0}}+d$ (Proposition 2.7).

From the formula for $S_{\text {cone }\left(A_{i} \cup B_{\tau}\right)}$ in Theorem 3.2 , it follows that

$$
S_{\text {cone }\left(A_{i} \cup B_{\tau}\right)}=\frac{\sum_{h \in H_{A_{i} \cup B_{\tau}}} p^{\sigma(h)+m(h) s}}{\prod_{x \in A_{i} \cup B_{\tau}}\left(p^{\sigma(x)+m(x) s}-1\right)} .
$$

We know that $m$ is linear on $\bar{\Delta}_{\tau}(f)$, and so $\sigma$ and $m$ are linear on $H_{A_{i} \cup B_{\tau}}$. It is also clear that $\sigma(a)+m(a) \rho=0$ for every $a \in$ cone $A_{i} \subset \bar{\Delta}_{\tau_{0}}(f)$. Let $\pi$ be the natural projection of vet $A_{i}+\operatorname{vct} B_{\tau}$ onto vet $B_{\tau}$. Then

$$
\begin{aligned}
\sum_{h \in H_{A_{i} \cup B_{\tau}}} p^{\sigma(h)+m(h) \rho} & =\sum_{h \in H_{A_{i} \cup B_{\tau}}} p^{\sigma(\pi(h))+m(\pi(h)) \rho} \\
& =\# H_{A_{i}} \sum_{b \in \pi\left(H_{A_{i} \cup B_{\tau}}\right)} p^{\sigma(b)+m(b) \rho},
\end{aligned}
$$

by Lemma $5.2(\mathrm{i})$. Consequently, $\lim _{s \rightarrow \rho}\left(p^{s-\rho}-1\right)^{\kappa} S_{\text {cone }\left(A_{i} \cup B_{\tau}\right)}$ equals

$$
\lim _{s \rightarrow \rho}\left(p^{s-\rho}-1\right)^{\kappa} \frac{\# H_{A_{i}}}{\prod_{x \in A_{i}}\left(p^{\sigma(x)+m(x) s}-1\right)} \frac{\sum_{b \in \pi\left(H_{\left.A_{i} \cup B_{\tau}\right)}\right.} p^{\sigma(b)+m(b) \rho}}{\prod_{x \in B_{\tau}}\left(p^{\sigma(x)+m(x) \rho}-1\right)} .
$$

To finish the proof, we define $S(P, V, g, s)$ for a finite subset $P$ of $\mathbb{Q}^{n}$, a finite subset $V$ of $\mathbb{N}^{n}$, a polynomial $g$ and $s \in \mathbb{C}$ as

$$
S(P, V, g, s)=\frac{\sum_{x \in P} p^{\sigma(x)+m(x) s}}{\prod_{x \in V}\left(p^{\sigma(x)+m(x) s}-1\right)},
$$

and where the function $m$ is completely determined by the Newton polyhedron $\Gamma(g)$ of the polynomial $g$. Note that if cone $V$ is a rational simplicial cone contained in $\left(\mathbb{R}^{+}\right)^{n}$ (i.e., $V$ is a finite subset of $\mathbb{N}^{n}$ such that its elements are linearly independent) and cone $V$ is contained in a cone associated to a face of $\Gamma(g)$, then

$$
S_{\text {cone } V}=S\left(H_{V}, V, g, s\right),
$$

where $S_{\text {cone } V}$ is defined as in Theorem 3.2 Using this notation,

$$
\lim _{s \rightarrow \rho}\left(p^{s-\rho}-1\right)^{\kappa} S_{\text {cone }}\left(A_{i} \cup B_{\tau}\right)
$$

equals

$$
S\left(\pi\left(H_{A_{i} \cup B_{\tau}}\right), B_{\tau}, f, \rho\right) \lim _{s \rightarrow \rho}\left(p^{s-\rho}-1\right)^{\kappa} S_{\text {cone } A_{i}} .
$$

Note that the sets $A_{i}$ all generate the same vector space. So, Lemma 5.2 ii) implies that $\pi\left(H_{A_{i} \cup B_{\tau}}\right)=\pi\left(H_{A_{1} \cup B_{\tau}}\right)$ for every $i \in\{1, \ldots, \ell\}$. Therefore,

$$
\begin{aligned}
& \lim _{s \rightarrow \rho}\left(p^{s-\rho}-1\right)^{\kappa} S_{\Delta_{\tau}(f)} \\
& =\sum_{B_{\tau}} \sum_{i=1}^{\ell} \lim _{s \rightarrow \rho}\left(p^{s-\rho}-1\right)^{\kappa} S_{\text {cone }\left(A_{i} \cup B_{\tau}\right)} \\
& =\left(\sum_{B_{\tau}} S\left(\pi\left(H_{A_{1} \cup B_{\tau}}\right), B_{\tau}, f, \rho\right)\right) \lim _{s \rightarrow \rho}\left(p^{s-\rho}-1\right)^{\kappa} S_{\Delta_{\tau_{0}}(f)},
\end{aligned}
$$


where $B_{\tau}$ runs through all the sets described in Assertion 5.4. Similarly,

$$
\begin{aligned}
& \lim _{s \rightarrow \rho}\left(p^{s-\rho}-1\right)^{\kappa} S_{\Delta_{\tau}\left(f_{\tau_{0}}\right)} \\
& \quad=\left(\sum_{B_{\tau}} S\left(\pi\left(H_{A_{1}^{\prime} \cup B_{\tau}}\right), B_{\tau}, f_{\tau_{0}}, \rho\right)\right) \lim _{s \rightarrow \rho}\left(p^{s-\rho}-1\right)^{\kappa} S_{\Delta_{\tau_{0}}\left(f_{\tau_{0}}\right)} .
\end{aligned}
$$

Note that $A_{1}^{\prime}$ generates the same vector space as $A_{1}$. So, the sets $\pi\left(H_{A_{1}^{\prime} \cup B_{\tau}}\right)$ and $\pi\left(H_{A_{1} \cup B_{\tau}}\right)$ are equal, by Lemma 5.2(ii). Moreover, the $m(b)$ for $b \in$ $\pi\left(H_{A_{1} \cup B_{\tau}}\right) \cup B_{\tau}$ are also the same, because $m(b)=b \cdot x_{\tau}$ for every $b \in \bar{\Delta}_{\tau}$, where $x_{\tau}$ is a fixed element of $\tau \subset \tau_{0}$. Now, it is clear that $S\left(\pi\left(H_{A_{1} \cup B_{\tau}}\right), B_{\tau}, f, \rho\right)=$ $S\left(\pi\left(H_{A_{1}^{\prime} \cup B_{\tau}}\right), B_{\tau}, f_{\tau_{0}}, \rho\right)$ and that

$$
\begin{aligned}
\frac{\lim _{s \rightarrow \rho}\left(p^{s-\rho}-1\right)^{\kappa} S_{\Delta_{\tau}(f)}}{\lim _{s \rightarrow \rho}\left(p^{s-\rho}-1\right)^{\kappa} S_{\Delta_{\tau_{0}}(f)}} & =\sum_{B_{\tau}} S\left(\pi\left(H_{A_{1} \cup B_{\tau}}\right), B_{\tau}, f, \rho\right) \\
& =\frac{\lim _{s \rightarrow \rho}\left(p^{s-\rho}-1\right)^{\kappa} S_{\Delta_{\tau}\left(f_{\tau_{0}}\right)}}{\lim _{s \rightarrow \rho}\left(p^{s-\rho}-1\right)^{\kappa} S_{\Delta_{\tau_{0}}\left(f_{\tau_{0}}\right)}}
\end{aligned}
$$

which proves (5.2).

One easily proves the following lemma.

Lemma 5.5. Suppose that $\Delta=$ cone $\left\{a_{1}, \ldots, a_{r}\right\}$ is the cone strictly positively spanned by the vectors $a_{1}, \ldots, a_{r} \in\left(\mathbb{R}^{+}\right)^{n} \backslash\{0\}$. Fix an element $b \in \Delta$. Then $\Delta$ is the disjoint union of the cones of the form

$$
W=\left\{\beta_{1} b+f \mid \beta_{1} \in \mathbb{R}, \beta_{1}>0, f \in \text { ri } F\right\},
$$

where $F$ is a proper face of $\bar{\Delta}$ and $\mathrm{ri} F$ is its interior relative to the affine hull of F.

\section{Checking if an arbitrary Candidate pole is a pole OF THE EXPECTED ORDER: REDUCTION FROM $f$ TO $f_{\mu}$ 'S}

Theorem 6.1 is a generalization of Theorem 5.3 to arbitrary candidate poles $s_{1}$ of $Z_{f}(s, \chi)$. Because the faces of $\Gamma(f)$ of maximal pure contribution to a candidate pole $s_{1}$ with $\operatorname{Re}\left(s_{1}\right) \neq \rho$ do not intersect the diagonal $D=\left\{(t, t, \ldots, t) \in \mathbb{R}^{n}\right\}$, the situation can be more difficult.

Theorem 6.1. Suppose that the prime number $p$, the polynomial $f$ and the character $\chi$ satisfy the conditions of Theorem 3.2. Suppose that $s_{1}$ is a candidate pole of $Z_{f}(s, \chi)$, i.e., a number from the set described in Proposition 4.1. Suppose that, for every face $\mu$ of $\Gamma(f)$ of maximal pure contribution to the candidate pole $s_{1}$ of $Z_{f}(s, \chi)$, the face $\mu$ is the only face of $\Gamma\left(f_{\mu}\right)$ of maximal pure contribution to the candidate pole $s_{1}$ of $Z_{f_{\mu}}(s, \chi)$. Then

$$
\lim _{s \rightarrow s_{1}}\left(p^{s-s_{1}}-1\right)^{k} Z_{f}(s, \chi)=\sum_{\mu} c_{\mu} \lim _{s \rightarrow s_{1}}\left(p^{s-s_{1}}-1\right)^{k} Z_{f_{\mu}}(s, \chi),
$$

where $\mu$ runs through the faces of $\Gamma(f)$ of maximal pure contribution to the candidate pole $s_{1}$,

$$
k= \begin{cases}\kappa\left(s_{1}\right)+1 & \text { if } p^{s_{1}+1}=1 \text { and } \chi=\chi_{\text {triv }} \\ \kappa\left(s_{1}\right) & \text { otherwise }\end{cases}
$$


and the $c_{\mu}>0$ are constant real numbers, independent of $p$. In fact,

$$
c_{\mu}=\frac{\operatorname{Vol}\left(\bar{\Delta}_{\mu}(f) \cap H_{1}^{-}\right)}{\operatorname{Vol}\left(\bar{\Delta}_{\mu}\left(f_{\mu}\right) \cap H_{1}^{-}\right)},
$$

where the volume is the one on the vector space generated by $\bar{\Delta}_{\mu}$, induced by the standard metric on $\mathbb{R}^{n}$ and $H_{1}^{-}$is the half-space $\left\{x \in \mathbb{R}^{n} \mid \sum_{i=1}^{n} x_{i} \leq 1\right\}$.

Remarks.

(1) Suppose that $\mu$ is a face of $\Gamma(f)$ of maximal pure contribution to the candidate pole $s_{1}$. If the dimension of $\mu$ is 0 or 1 , then $\mu$ is the only face of $\Gamma\left(f_{\mu}\right)$ of maximal pure contribution to the candidate pole $s_{1}$ of $Z_{f_{\mu}}(s, \chi)$. If the dimension of $\mu$ is $>1$, the condition on $\mu$ will be satisfied in most examples. Nevertheless, it is possible that $\mu$ is not the only face of $\Gamma\left(f_{\mu}\right)$ of maximal pure contribution to the candidate pole $s_{1}$. For example, let $f(x, y, z)=x^{7} y^{3} z+x^{4} y^{8}+x^{8} y^{4}+z^{7}$. Then the Newton polyhedron $\Gamma(f)$ is given by the system of linear inequalities

$$
\left\{\begin{array}{l}
x \geq 0 \\
y \geq 0 \\
z \geq 0 \\
x+y+2 z \geq 12 \\
y+z \geq 4 \\
2 y+z \geq 7 \\
27 x+25 y+44 z \geq 308 \\
7 x+4 z \geq 28
\end{array}\right.
$$

(see also Figure 6.1). We denote the facets with supporting hyperplanes given by the corresponding equalities in the inequalities above by $\tau_{1}, \ldots, \tau_{8}$. Face $\tau_{4}$ is the only face of $\Gamma(f)$ of maximal pure contribution to the candidate pole $-1 / 3$.

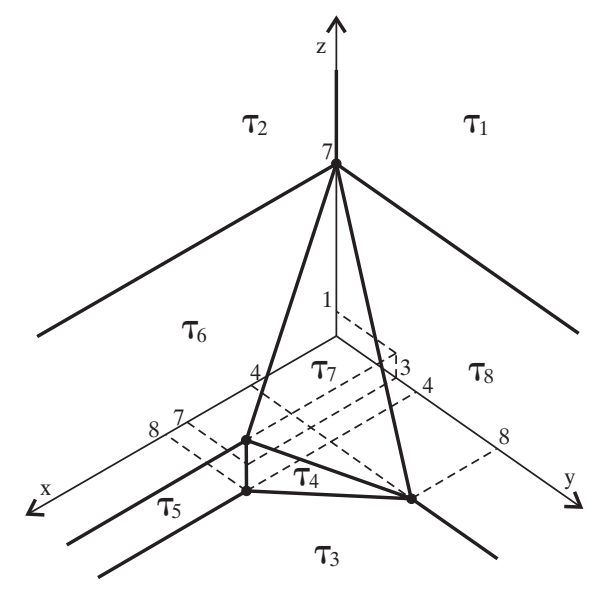

Figure 6.1. Newton polyhedron of $x^{7} y^{3} z+x^{4} y^{8}+x^{8} y^{4}+z^{7}$ 


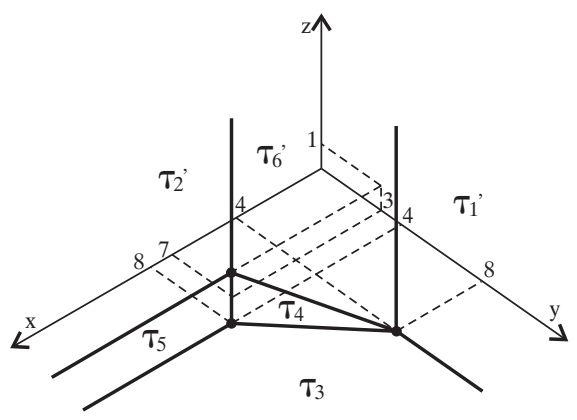

Figure 6.2. Newton polyhedron of $x^{7} y^{3} z+x^{4} y^{8}+x^{8} y^{4}$

The Newton polyhedron $\Gamma\left(f_{\tau_{4}}\right)$ of $f_{\tau_{4}}=x^{7} y^{3} z+x^{4} y^{8}+x^{8} y^{4}$ is given by the system of linear inequalities

$$
\left\{\begin{array}{l}
x \geq 4 \\
y \geq 3 \\
z \geq 0 \\
x+y+2 z \geq 12 \\
y+z \geq 4 \\
5 x+3 y \geq 44
\end{array}\right.
$$

(see also Figure 6.2). We denote the facets with supporting hyperplanes given by the corresponding equalities in the inequalities above by $\tau_{1}^{\prime}, \tau_{2}^{\prime}, \tau_{3}$, $\tau_{4}, \tau_{5}, \tau_{6}^{\prime}$. The face $\tau_{4}$ is not the only face of $\Gamma\left(f_{4}\right)$ of maximal pure contribution to the candidate pole $-1 / 3: \tau_{2}^{\prime}$ is also such a face.

(2) The proof below also shows that a similar result holds for $Z_{f, 0}(s, \chi)$, even if the faces of $\Gamma(f)$ of maximal pure contribution to the candidate pole $s_{1}$ are not compact. Then $f$ has to be non-degenerate over $\mathbb{F}_{p}$ with respect to all the compact faces of its Newton polyhedron.

Proof. By the same argument as in the proof of Theorem 5.3 we can also show that $f_{\mu}$ is non-degenerate over the finite field $\mathbb{F}_{p}$ with respect to all the faces of its Newton polyhedron $\Gamma\left(f_{\mu}\right)$ if $\mu$ is a face of $\Gamma(f)$. So, we can use the formulas for $Z_{f}(s, \chi)$ and $Z_{f_{\mu}}(s, \chi)$ from Theorem 3.2

We know from [Hoo02, Proposition 4.9] that $\lim _{s \rightarrow s_{1}}\left(p^{s-s_{1}}-1\right)^{\kappa\left(s_{1}\right)} S_{\Delta_{\tau}}=0$ for faces $\tau$ not contained in any face $\mu$ of $\Gamma(f)$ of maximal pure contribution to the candidate pole $s_{1}$. It is clear that $L_{\tau}(f)=L_{\tau}\left(f_{\mu}\right)$ for faces $\tau$ contained in a face $\mu$ of $\Gamma(f)$. For faces $\tau$ contained in at least one face $\mu$ of $\Gamma(f)$ of maximal pure contribution to the candidate pole, we will write

$$
\lim _{s \rightarrow s_{1}}\left(p^{s-s_{1}}-1\right)^{\kappa\left(s_{1}\right)} S_{\Delta_{\tau}(f)}=\sum_{\mu} R_{\mu},
$$

where $\mu$ runs through all the faces of $\Gamma(f)$ of maximal pure contribution to the candidate pole $s_{1}$ and where the $R_{\mu}$, which depend on $\tau$, will be specified later. The $R_{\mu}$ for $\mu \not \supset \tau$ will vanish. It suffices then to show that

$$
\frac{R_{\mu}}{\lim _{s \rightarrow s_{1}}\left(p^{s-s_{1}}-1\right)^{\kappa\left(s_{1}\right)} S_{\Delta_{\mu}(f)}}=\frac{\lim _{s \rightarrow s_{1}}\left(p^{s-s_{1}}-1\right)^{\kappa\left(s_{1}\right)} S_{\Delta_{\tau}\left(f_{\mu}\right)}}{\lim _{s \rightarrow s_{1}}\left(p^{s-s_{1}}-1\right)^{\kappa\left(s_{1}\right)} S_{\Delta_{\mu}\left(f_{\mu}\right)}}
$$


for faces $\tau \subset \mu$, and that

$$
\frac{\lim _{s \rightarrow s_{1}}\left(p^{s-s_{1}}-1\right)^{\kappa\left(s_{1}\right)} S_{\Delta_{\mu}(f)}}{\lim _{s \rightarrow s_{1}}\left(p^{s-s_{1}}-1\right)^{\kappa\left(s_{1}\right)} S_{\Delta_{\mu}\left(f_{\mu}\right)}}=\frac{\operatorname{Vol}\left(\bar{\Delta}_{\mu}(f) \cap H_{1}^{-}\right)}{\operatorname{Vol}\left(\bar{\Delta}_{\mu}\left(f_{\mu}\right) \cap H_{1}^{-}\right)},
$$

where $\mu$ is a face of $\Gamma(f)$ of maximal pure contribution to the candidate pole $s_{1}$.

The last equality follows by Proposition 4.7 and the fact that $\bar{\Delta}_{\mu}(f)$ and $\bar{\Delta}_{\mu}\left(f_{\mu}\right)$ generate the same vector space.

Suppose that $\bigcup_{i} \Delta_{i}$ is a finite partition of $\Delta_{\tau}$ into rational simplicial cones such that each $\Delta_{i}$ has at most $\kappa\left(s_{1}\right)$ generators contributing to the candidate pole $s_{1}$. This is always possible; see below. Then $S_{\Delta_{\tau}}=\sum_{i} S_{\Delta_{i}}$. From the formula for $S_{\Delta_{i}}$ in Theorem 3.2, it follows that $\lim _{s \rightarrow s_{1}}\left(p^{s-s_{1}}-1\right)^{\kappa\left(s_{1}\right)} S_{\Delta_{i}}=0$ if less than $\kappa\left(s_{1}\right)$ generators of $\Delta_{i}$ contribute to the candidate pole $s_{1}$. So, to prove equality (6.2), it is only important to know the other cones. We will choose partitions of $\Delta_{\tau}(f)$ and $\Delta_{\tau}\left(f_{\mu}\right)$ that are closely related to some chosen partitions of $\Delta_{\mu}(f)$ and $\Delta_{\mu}\left(f_{\mu}\right)$, respectively.

Fix a finite partition of $\Delta_{\mu}(f)$ into rational simplicial cones, and let

$$
\text { cone } A_{i, \mu} \text {, for } i=1, \ldots, \ell_{\mu} \text {, }
$$

be the only cones of dimension $\kappa\left(s_{1}\right)$, i.e., with $\kappa\left(s_{1}\right)$ generators contributing to the candidate pole $s_{1}$, in this partition. Here, each $A_{i, \mu}$ is a set of $\kappa\left(s_{1}\right)$ vectors in $\mathbb{N}^{n}$ that are linearly independent over $\mathbb{Q}$. Similarly, fix a finite partition of $\Delta_{\mu}\left(f_{\mu}\right)$ into rational simplicial cones, and let

$$
\text { cone } A_{j, \mu}^{\prime}, \text { for } j=1, \ldots, m_{\mu},
$$

be the only cones of dimension $\kappa\left(s_{1}\right)$ in this partition where each $A_{j, \mu}^{\prime}$ is a set of $\kappa\left(s_{1}\right)$ vectors in $\mathbb{N}^{n}$ that are linearly independent over $\mathbb{Q}$.

It is clear that $\Delta_{\tau}(f) \subset \Delta_{\tau}\left(f_{\mu}\right)$ for every face $\tau \subset \mu$. Now for each face $\tau \varsubsetneqq \mu$ fix a primitive vector

$$
b_{\tau} \in \Delta_{\tau}(f) \subset \Delta_{\tau}\left(f_{\mu}\right)
$$

that does not contribute to the candidate pole $s_{1}$. This is always possible because $\sigma$ and $m$ are linear on $\bar{\Delta}_{\tau}(f)$ and not all generators of $\Delta_{\tau}(f)$ contribute to the candidate pole $s_{1}$. From Lemma 2.5 and Lemma 5.5. it follows by induction on $d$ that the following assertion is true for any $d$ in $\mathbb{N} \backslash\{0\}$.

Assertion 6.2. Suppose that the face $\tau$ is a face of $\Gamma(f)$ that is contained in at least one face of $\Gamma(f)$ of maximal pure contribution to the candidate pole $s_{1}$ and that $\operatorname{dim} \tau=n-\kappa\left(s_{1}\right)-d$. Then there exists a partition of $\Delta_{\tau}(f)$ into rational simplicial cones such that the only cones in this partition that have $\kappa\left(s_{1}\right)$ generators contributing to the candidate pole $s_{1}$ are precisely the cones of the form

$$
\text { cone }\left(A_{i, \mu} \cup B_{\tau}\right) \text {, }
$$

where $\mu \supset \tau$ is a face of $\Gamma(f)$ of maximal pure contribution to the candidate pole $s_{1}, i \in\left\{1, \ldots, \ell_{\mu}\right\}$, and $B_{\tau}$ is a set of the form $\left\{b_{\tau_{1}}, \ldots, b_{\tau_{v-1}}, b_{\tau}\right\}$, where $v \leq d$ and $v=d$ for the cones in this partition of dimension equal to $\operatorname{dim} \Delta_{\tau}(f), \tau \varsubsetneqq \tau_{v-1} \varsubsetneqq$ $\cdots \varsubsetneqq \tau_{1} \varsubsetneqq \mu$ are faces of $\mu$ and $b_{\tau_{1}}, \ldots, b_{\tau_{v-1}}, b_{\tau}$ are defined in (6.4). The other cones in the partition have less than $\kappa\left(s_{1}\right)$ generators contributing to the candidate pole $s_{1}$. Similarly, there exists a partition of $\Delta_{\tau}\left(f_{\mu}\right)$ into rational simplicial cones 
such that the only cones in this partition that have $\kappa\left(s_{1}\right)$ generators contributing to the candidate pole $s_{1}$ are precisely the cones of the form

$$
\text { cone }\left(A_{j, \mu}^{\prime} \cup B_{\tau}\right) \text {, }
$$

where $j \in\left\{1, \ldots, m_{\mu}\right\}$ and $B_{\tau}$ is defined as above. The other cones in the partition have less than $\kappa\left(s_{1}\right)$ generators contributing to the candidate pole $s_{1}$.

From the formula for $S_{\text {cone }\left(A_{i, \mu} \cup B_{\tau}\right)}$ in Theorem 3.2 it follows that

$$
S_{\text {cone }\left(A_{i, \mu} \cup B_{\tau}\right)}=\frac{\sum_{h \in H_{A_{i, \mu} \cup B_{\tau}}} p^{\sigma(h)+m(h) s}}{\prod_{x \in A_{i, \mu} \cup B_{\tau}}\left(p^{\sigma(x)+m(x) s}-1\right)} .
$$

We know that $m$ is linear on $\bar{\Delta}_{\tau}$, and so $\sigma$ and $m$ are linear on $H_{A_{i, \mu} \cup B_{\tau}}$. It is also clear that $p^{\sigma(a)+m(a) s_{1}}=1$ for every $a \in$ cone $A_{i, \mu} \subset \bar{\Delta}_{\mu}(f)$ because all generators of $\Delta_{\mu}(f)$ contribute to the candidate pole $s_{1}$. Let $\pi$ be the natural projection of vct $A_{i, \mu}+\operatorname{vct} B_{\tau}$ onto vct $B_{\tau}$. Then

$$
\begin{aligned}
\sum_{h \in H_{A_{i, \mu} \cup B_{\tau}}} p^{\sigma(h)+m(h) s_{1}} & =\sum_{h \in H_{A_{i, \mu} \cup B_{\tau}}} p^{\sigma(\pi(h))+m(\pi(h)) s_{1}} \\
& =\# H_{A_{i, \mu}} \sum_{b \in \pi\left(H_{A_{i, \mu} \cup B_{\tau}}\right)} p^{\sigma(b)+m(b) s_{1}},
\end{aligned}
$$

by Lemma $\left[5.2(\mathrm{i})\right.$. Consequently, $\lim _{s \rightarrow s_{1}}\left(p^{s-s_{1}}-1\right)^{\kappa\left(s_{1}\right)} S_{\text {cone }\left(A_{i, \mu} \cup B_{\tau}\right)}$ equals

$$
\lim _{s \rightarrow s_{1}}\left(p^{s-s_{1}}-1\right)^{\kappa\left(s_{1}\right)} \frac{\# H_{A_{i, \mu}}}{\prod_{x \in A_{i, \mu}}\left(p^{\sigma(x)+m(x) s}-1\right)} \frac{\sum_{b \in \pi\left(H_{A_{i, \mu} \cup B_{\tau}}\right)} p^{\sigma(b)+m(b) s_{1}}}{\prod_{x \in B_{\tau}}\left(p^{\sigma(x)+m(x) s_{1}}-1\right)} .
$$

Using the same notation as in the proof of Theorem 5.3 we have

$$
\begin{aligned}
& \lim _{s \rightarrow s_{1}}\left(p^{s-s_{1}}-1\right)^{\kappa\left(s_{1}\right)} S_{\text {cone }\left(A_{i, \mu} \cup B_{\tau}\right)} \\
& \quad=S\left(\pi\left(H_{A_{i, \mu} \cup B_{\tau}}\right), B_{\tau}, f, s_{1}\right) \lim _{s \rightarrow s_{1}}\left(p^{s-s_{1}}-1\right)^{\kappa\left(s_{1}\right)} S_{\text {cone } A_{i, \mu}} .
\end{aligned}
$$

Note that the sets $A_{i, \mu}$ all generate the same vector space. So, Lemma 5.2 (ii) implies that $\pi\left(H_{A_{i, \mu} \cup B_{\tau}}\right)=\pi\left(H_{A_{1, \mu} \cup B_{\tau}}\right)$ for every $i \in\left\{1, \ldots, \ell_{\mu}\right\}$. Therefore,

$$
\lim _{s \rightarrow s_{1}}\left(p^{s-s_{1}}-1\right)^{\kappa\left(s_{1}\right)} S_{\Delta_{\tau}(f)}=\sum_{\mu} R_{\mu},
$$

where $\mu$ runs through the faces of $\Gamma(f)$ of maximal pure contribution to the candidate pole $s_{1}$ (that contain $\tau$ ) and

$$
\begin{aligned}
R_{\mu} & =\sum_{B_{\tau}} \sum_{i=1}^{\ell_{\mu}} \lim _{s \rightarrow s_{1}}\left(p^{s-s_{1}}-1\right)^{\kappa\left(s_{1}\right)} S_{\text {cone }\left(A_{i, \mu} \cup B_{\tau}\right)} \\
& =\left(\sum_{B_{\tau}} S\left(\pi\left(H_{A_{1, \mu} \cup B_{\tau}}\right), B_{\tau}, f, s_{1}\right)\right) \lim _{s \rightarrow s_{1}}\left(p^{s-s_{1}}-1\right)^{\kappa\left(s_{1}\right)} S_{\Delta_{\mu}(f)},
\end{aligned}
$$


where $B_{\tau}$ runs through all the sets described in Assertion 6.2. Similarly, for $\mu$ a fixed face of $\Gamma(f)$ of maximal pure contribution to the candidate pole $s_{1}$, we have

$$
\begin{aligned}
& \lim _{s \rightarrow s_{1}}\left(p^{s-s_{1}}-1\right)^{\kappa\left(s_{1}\right)} S_{\Delta_{\tau}\left(f_{\mu}\right)} \\
& \quad=\left(\sum_{B_{\tau}} S\left(\pi\left(H_{A_{1, \mu}^{\prime} \cup B_{\tau}}\right), B_{\tau}, f_{\mu}, s_{1}\right)\right) \lim _{s \rightarrow s_{1}}\left(p^{s-s_{1}}-1\right)^{\kappa\left(s_{1}\right)} S_{\Delta_{\mu}\left(f_{\mu}\right),}
\end{aligned}
$$

because $\mu$ is the only face of $\Gamma\left(f_{\mu}\right)$ of maximal pure contribution to the candidate pole $s_{1}$.

Note that $A_{1, \mu}^{\prime}$ generates the same vector space as $A_{1, \mu}$. So, the sets $\pi\left(H_{A_{1, \mu}^{\prime} \cup B_{\tau}}\right)$ and $\pi\left(H_{A_{1, \mu} \cup B_{\tau}}\right)$ are equal, by Lemma 5.2 (ii). Moreover, for $b \in \pi\left(H_{A_{1, \mu} \cup B_{\tau}}\right) \cup B_{\tau}$, the $m(b)$ are also the same because $m(b)=b \cdot x_{\tau}$ for every $b \in \bar{\Delta}_{\tau}$, where $x_{\tau}$ is a fixed element of $\tau \subset \mu$. Now, it is clear that $S\left(\pi\left(H_{A_{1, \mu} \cup B_{\tau}}\right), B_{\tau}, f, s_{1}\right)=$ $S\left(\pi\left(H_{A_{1}^{\prime} \cup B_{\tau}}\right), B_{\tau}, f_{\mu}, s_{1}\right)$ and that

$$
\begin{aligned}
\frac{R_{\mu}}{\lim _{s \rightarrow s_{1}}\left(p^{s-s_{1}}-1\right)^{\kappa\left(s_{1}\right)} S_{\Delta_{\mu}(f)}} & =\sum_{B_{\tau}} S\left(\pi\left(H_{A_{1, \mu} \cup B_{\tau}}\right), B_{\tau}, f, s_{1}\right) \\
& =\frac{\lim _{s \rightarrow s_{1}}\left(p^{s-s_{1}}-1\right)^{\kappa\left(s_{1}\right)} S_{\Delta_{\tau}\left(f_{\mu}\right)}}{\lim _{s \rightarrow s_{1}}\left(p^{s-s_{1}}-1\right)^{\kappa\left(s_{1}\right)} S_{\Delta_{\mu}\left(f_{\mu}\right)}},
\end{aligned}
$$

which proves 6.2 .

7. Another explicit Formula for $Z_{f}(s, \chi)$ When $f=f_{\sigma}$, WHERE $\sigma \subset \Gamma(f)$ IS AN UNSTABLE CONVEX SET,

AND PRoOf of the CONJeCture of Denef and SARgos

We refer to [DS92 for an explanation of the choice of the name "unstable", which is used in Definition 7.1 In contrast to [DS92, we do not only define the notion of an unstable face of $\Gamma(f)$, but also the notion of an unstable convex set. Moreover, for the following results, it is not necessary to exclude the case where $\sigma \subset\left\{\left(x_{1}, \ldots, x_{n}\right) \in \mathbb{R}^{n} \mid x_{j}=0\right\}$. Of course, the face $\tau_{0}$ cannot be contained in the set $\left\{\left(x_{1}, \ldots, x_{n}\right) \in \mathbb{R}^{n} \mid x_{j}=0\right\}$ because $\tau_{0}$ must intersect the diagonal and the origin does not belong to the Newton polyhedron.

Definition 7.1. Let $f$ be as in Definition 1.2, and let $p$ be a prime number. A convex set $\sigma$ is called unstable over $\mathbb{F}_{p}$ relative to $x_{j}$ and with respect to $f$, if the following two conditions are satisfied:

(i) $\sigma \subset\left\{\left(x_{1}, \ldots, x_{n}\right) \in \mathbb{R}^{n} \mid 0 \leq x_{j} \leq 1\right\}$ and

(ii) for every face $\tau$ of $\sigma$ contained in $\left\{\left(x_{1}, \ldots, x_{n}\right) \in \mathbb{R}^{n} \mid x_{j}=1\right\}$, the polynomial $\bar{f}_{\tau}$ does not vanish on $\left(\mathbb{F}_{p}^{\times}\right)^{n}$.

A convex set $\sigma$ is called unstable over $\mathbb{F}_{p}$ with respect to $f$ if there exists an index $j(1 \leq j \leq n)$ such that $\sigma$ is unstable over $\mathbb{F}_{p}$ relative to $x_{j}$ and with respect to $f$.

We have a similar definition for a convex set $\sigma$ that is unstable over $\mathbb{C}$ with respect to $f$. Then $f$ has to be a polynomial over $\mathbb{C}$, and in (ii) the polynomial $f_{\tau}$ does not vanish on $\left(\mathbb{C}^{\times}\right)^{n}$.

In Theorem 7.3 we will give a formula for $Z_{f}(s, \chi)$ that holds if $f=f_{\sigma}$ where $\sigma \subset \Gamma(f)$ is an unstable convex set over $\mathbb{F}_{p}$ with respect to $f$ and if $f$ is nondegenerate over $\mathbb{F}_{p}$ with respect to a particular set of faces of $\Gamma(f)$. This formula 
will be similar to the one in Theorem 3.2 But first, we state Lemma 7.2 and give the definition of a direction of recession.

Lemma 7.2. Let $p$ be a prime number and $f(x)=f\left(x_{1}, \ldots, x_{n}\right) \in \mathbb{Z}_{p}\left[x_{1}, \ldots, x_{n}\right]$. Indicate by $\bar{f}$ the polynomial over $\mathbb{F}_{p}$ obtained by reducing each coefficient of $f$ modulo $p \mathbb{Z}_{p}$. Let $s$ be a complex variable with $\operatorname{Re}(s)>0$, and let $\chi$ be a character of $\mathbb{Z}_{p}^{\times}$with conductor $c_{\chi}$. Let $\tilde{N}$ be the number of elements in the set $\left\{a \in\left(\mathbb{F}_{p}^{\times}\right)^{n-1} \times\right.$ $\left.\mathbb{F}_{p} \mid \bar{f}(a)=0\right\}$. Suppose that the set of congruences

$$
\left\{\begin{array}{l}
f_{\tau}(x) \equiv 0 \bmod p, \\
\frac{\partial f_{\tau}}{\partial x_{i}}(x) \equiv 0 \bmod p, \quad i=1, \ldots, n,
\end{array}\right.
$$

has no solution in $\left(\mathbb{Z}_{p}^{\times}\right)^{n-1} \times \mathbb{Z}_{p}$. Then one has

$$
\begin{aligned}
& \int \chi(\operatorname{ac} f(x))|f(x)|^{s}|d x| \\
& =\left\{\begin{array}{cc}
p^{-n}\left((p-1)^{n-1} p-p \tilde{N} \frac{p^{s}-1}{p^{s+1}-1}\right) & \text { for } \chi=\chi_{\text {triv }}, \\
p^{-n c_{\chi}} \sum_{\left(\mathbb{Z}_{p}^{\times}\right)^{n-1} \times \mathbb{Z}_{p}} \chi(f(a)) & \text { for } \chi \neq \chi_{\text {triv }} . \\
& \begin{array}{l}
\left.a \in \mathbb{Z}_{p}^{\times}\right)^{n-1} \times \mathbb{Z}_{p} \\
\text { mod } p^{c} \chi \\
f(a) \neq \equiv \bmod p
\end{array}
\end{array}\right.
\end{aligned}
$$

Proof. The proof is similar to the proof of [DH01, Corollary 3.2] and [Hoo02, Proposition 3.2].

Let $\tau$ be a face of the Newton polyhedron of a polynomial $f$ and $e_{i}$ the $i$-th unit vector of $\mathbb{R}^{n}$. We call $e_{i}$ a direction of recession of $\tau$ if $\tau$ recedes in the direction of $e_{i}$, i.e.,

$$
\forall x \in \tau, \forall \lambda \in \mathbb{R} \text { with } \lambda \geq 0: x+\lambda e_{i} \in \tau .
$$

Theorem 7.3. Let $f$ be as in Definition 1.2, and let $p$ be a prime number. Suppose that $f$ is non-degenerate over $\mathbb{F}_{p}$ with respect to all the faces of $\Gamma(f)$ contained in $\left\{\left(x_{1}, \ldots, x_{n}\right) \in \mathbb{R}^{n} \mid x_{n}=0\right\}$ and suppose that $f=f_{\sigma}$ where $\sigma \subset \Gamma(f)$ is an unstable convex set over $\mathbb{F}_{p}$ relative to $x_{n}$ and with respect to $f$. Let $s$ be a complex variable with $\operatorname{Re}(s)>0$, and let $\chi$ be a character of $\mathbb{Z}_{p}^{\times}$with conductor $c_{\chi}=1$. Then

$$
Z_{f}(s, \chi)=\sum_{\substack{\tau \text { face of } \Gamma(f) \\ e_{n} \in \operatorname{rec} \tau}} \tilde{L}_{\tau} S_{\Delta_{\tau}}
$$

with

$$
\begin{aligned}
& \tilde{L}_{\tau}= \begin{cases}p^{-n}\left((p-1)^{n-1} p-p \tilde{N}_{\tau} \frac{p^{s}-1}{p^{s+1}-1}\right) & \text { for } \chi=\chi_{\text {triv }}, \\
p^{-n} \sum_{a \in\left(\mathbb{F}_{p}^{\times}\right)^{n-1} \times \mathbb{F}_{p}} \chi\left(f_{\tau}(a)\right) & \text { for } \chi \neq \chi_{\text {triv }},\end{cases} \\
& \tilde{N}_{\tau}=\left\{a \in\left(\mathbb{F}_{p}^{\times}\right)^{n-1} \times \mathbb{F}_{p} \mid \bar{f}_{\tau}(a)=0\right\},
\end{aligned}
$$

$S_{\Delta_{\tau}}$ as in Theorem [3.2, $e_{n}=(0,0, \ldots, 1)$ and $\operatorname{rec} \tau$ the set of directions of recession of $\tau$. Recall that $\Gamma(f)$ is also a face of $\Gamma(f)$ with $e_{n}$ as direction of recession.

Moreover, suppose that $s_{1}$ is a candidate pole of $Z_{f}(s, \chi)$, i.e., a number from the set described in Proposition 4.1. If $e_{n} \notin \operatorname{rec} \mu$ for each face $\mu$ of $\Gamma(f)$ of maximal pure contribution to the candidate pole $s_{1}$, then the following holds: If $p^{s_{1}+1} \neq 1$ or 
$\chi \neq \chi_{\text {triv }}$ and $s_{1}$ is a pole of $Z_{f}(s, \chi)$, its order is $<\kappa\left(s_{1}\right)$; if $p^{s_{1}+1}=1$ and $s_{1}$ is a pole of $Z_{f}(s)$, its order is $<\kappa\left(s_{1}\right)+1$.

Remarks.

(1) Note that formula (7.1) holds if $f$ is non-degenerate over $\mathbb{F}_{p}$ with respect to all the faces of $\Gamma(f)$ contained in $\left\{\left(x_{1}, \ldots, x_{n}\right) \in \mathbb{R}^{n} \mid x_{n}=0\right\}$ and if $\Gamma_{\mathrm{gl}}(f)$ is an unstable convex set over $\mathbb{F}_{p}$ relative to $x_{n}$ and with respect to $f$.

The condition " $f=f_{\sigma}$ with $\sigma \subset \Gamma(f)$ an unstable convex set over $\mathbb{F}_{p}$ with respect to $f$ " is in fact too strong. It is already enough that there exists a convex set $\sigma$ such that the following holds: for each face $\tau$ of $\Gamma(f)$ with $e_{n}$ a direction of recession, the polynomial $\bar{f}_{\sigma \cap \tau \cap H_{1}}$ does not vanish on $\left(\mathbb{F}_{p}^{\times}\right)^{n}$, with $H_{1}=\left\{\left(x_{1}, \ldots, x_{n}\right) \in \mathbb{R}^{n} \mid x_{n}=1\right\}$. Moreover, the proof below shows that formula (7.1) for $Z_{f}(s, \chi)$ is even true when one has, for every face $\tau$ of $\Gamma(f)$ for which $e_{n}$ is a direction of recession, that the set of congruences

$$
\left\{\begin{array}{l}
f_{\tau}(x) \equiv 0 \bmod p \\
\frac{\partial f_{\tau}(x)}{\partial x_{i}} \equiv 0 \bmod p, \quad i=1, \ldots, n
\end{array}\right.
$$

has no solution in $\left(\mathbb{Z}_{p}^{\times}\right)^{n-1} \times \mathbb{Z}_{p}$.

(2) Let $f(x)$ be a non-zero polynomial over $\mathbb{Z}$ with $f(0)=0$. Suppose that $f$ is non-degenerate over $\mathbb{C}$ with respect to all the faces of $\Gamma(f)$ contained in $\left\{\left(x_{1}, \ldots, x_{n}\right) \in \mathbb{R}^{n} \mid x_{n}=0\right\}$ and suppose that $f=f_{\sigma}$ where $\sigma \subset \Gamma(f)$ is an unstable convex set over $\mathbb{C}$ relative to $x_{n}$ and with respect to $f$. Then the conditions of Theorem 7.3 are satisfied for $p \gg 0$.

Proof. Put $H_{0}=\left\{\left(x_{1}, \ldots, x_{n}\right) \in \mathbb{R}^{n} \mid x_{n}=0\right\}$ and $H_{1}=\left\{\left(x_{1}, \ldots, x_{n}\right) \in \mathbb{R}^{n} \mid x_{n}=\right.$ $1\}$. Note that the cones associated to the faces of $\Gamma(f)$ form a partition of $\left(\mathbb{R}^{+}\right)^{n}$. Moreover, for every $\left(a_{1}, \ldots, a_{n}\right) \in\left(\mathbb{R}^{+}\right)^{n}$, one has $a_{n}=0$ if and only if $e_{n}$ is a direction of recession of $F\left(a_{1}, \ldots, a_{n}\right)$. So, if $\Delta_{\tau} \cap H_{0} \neq \emptyset$, then $\Delta_{\tau}=\left\{a \in\left(\mathbb{R}^{+}\right)^{n} \mid\right.$ $F(a)=\tau\}$ is contained in $H_{0}$. Consequently, $H_{0} \cap\left(\mathbb{R}^{+}\right)^{n}$ is equal to the following disjoint union:

$$
\bigcup_{\tau \text { face of } \Gamma(f)}\left(\Delta_{\tau} \cap H_{0}\right)=\bigcup_{\substack{\tau \text { face of } \Gamma(f) \\ \Delta_{\tau} \subset H_{0}}} \Delta_{\tau}=\bigcup_{\substack{\tau \text { face of } \Gamma(f) \\ e_{n} \in \operatorname{rec} \tau}} \Delta_{\tau} .
$$

Therefore, $Z_{f}(s, \chi)$ equals

$$
\begin{aligned}
& \sum_{\left(k_{1}, \ldots, k_{n-1}\right) \in \mathbb{N}^{n-1}} \int_{\substack{\left(x_{1}, \ldots, x_{n}\right) \in \mathbb{Z}_{p}^{n} \\
\operatorname{ord}\left(x_{1}, \ldots, x_{n-1}\right)=\left(k_{1}, \ldots, k_{n-1}\right)}} \chi(\operatorname{ac} f(x))|f(x)|^{s}|d x| \\
& =\sum_{\substack{\tau \text { face of } \Gamma(f) \\
e_{n} \in \operatorname{rec} \tau}} \sum_{\left(k_{1}, \ldots, k_{n}-1,0\right) \in \mathbb{N}^{n} \cap \Delta_{\tau}} \\
& \int_{\substack{\left(x_{1}, \ldots, x_{n}\right) \in \mathbb{Z}_{p}^{n} \\
\operatorname{ord}\left(x_{1}, \ldots, x_{n-1}\right)=\left(k_{1}, \ldots, k_{n-1}\right)}} \chi(\operatorname{ac} f(x))|f(x)|^{s}|d x| .
\end{aligned}
$$


Suppose that $\tau$ is a face of $\Gamma(f)$ such that $e_{n}$ is a direction of recession of $\tau$ (or equivalently $\left.\Delta_{\tau} \subset H_{0}\right)$. Suppose that $k=\left(k_{1}, \ldots, k_{n-1}, 0\right) \in \mathbb{N}^{n} \cap \Delta_{\tau}$ and $x=$ $\left(x_{1}, \ldots, x_{n}\right) \in \mathbb{Z}_{p}^{n}$ with ord $\left(x_{1}, \ldots, x_{n-1}\right)=\left(k_{1}, \ldots, k_{n-1}\right)$. Write $x_{j}=p^{k_{j}} u_{j}$ with $u_{j} \in \mathbb{Z}_{p}^{\times}$, for $j=1, \ldots, n-1$. Then

$$
\begin{aligned}
|d x| & =p^{-\sigma(k)}|d u|\left|d x_{n}\right| \text { and } \\
x^{\omega} & =x_{1}^{\omega_{1}} \cdots x_{n-1}^{\omega_{n-1}} x_{n}^{\omega_{n}} \\
& =p^{\left(k_{1}, \ldots, k_{n-1}\right) \cdot\left(\omega_{1}, \ldots, \omega_{n-1}\right)} u_{1}^{\omega_{1}} \cdots u_{n-1}^{\omega_{n-1}} x_{n}^{\omega_{n}} \\
& =p^{k \cdot \omega} u_{1}^{\omega_{1}} \cdots u_{n-1}^{\omega_{n-1}} x_{n}^{\omega_{n}} .
\end{aligned}
$$

We know that $F(k)=\tau$. Thus $k \cdot \omega=m(k)$ for every $\omega \in \operatorname{supp}(f) \cap \tau$, and $k \cdot \omega \geq m(k)+1$ for every $\omega \in \operatorname{supp}(f) \backslash \tau$. It follows then that

$$
f(x)=p^{m(k)}\left(f_{\tau}\left(u_{1}, \ldots, u_{n-1}, x_{n}\right)+p \tilde{f}_{\tau, k}\left(u_{1}, \ldots, u_{n-1}, x_{n}\right)\right),
$$

where $\tilde{f}_{\tau, k}$ is a polynomial in the variables $u_{1}, \ldots, u_{n-1}, x_{n}$ over $\mathbb{Z}_{p}$ (depending on $\tau, k$ and $f)$. Hence, $Z_{f}(s, \chi)$ equals

$$
\begin{aligned}
& \sum_{\substack{\tau \text { face of } \Gamma(f) \\
e_{n} \in \operatorname{rec} \tau}} \sum_{\substack{k \in \mathbb{N}^{n} \cap \Delta_{\tau} \\
\left(\mathbb{Z}_{p}^{\times}\right)^{n-1} \times \mathbb{Z}_{p}}} \chi\left(\operatorname{ac}\left(f_{\tau}\left(u_{1}, \ldots, u_{n-1}, x_{n}\right)+p \tilde{f}_{\tau, k}\left(u_{1}, \ldots, u_{n-1}, x_{n}\right)\right)\right) \\
& \qquad\left|f_{\tau}\left(u_{1}, \ldots, u_{n-1}, x_{n}\right)+p \tilde{f}_{\tau, k}\left(u_{1}, \ldots, u_{n-1}, x_{n}\right)\right|^{s}|d u||d x| .
\end{aligned}
$$

Denote the last integral by $\tilde{L}_{\tau}$. By the following assertion and Lemma 7.2, we obtain equality (7.2) of Theorem 7.3

Assertion 7.4. For every face $\tau$ of $\Gamma(f)$ with $e_{n} \in \operatorname{rec} \tau$, one has that the set of congruences

$$
\left\{\begin{array}{l}
f_{\tau}(x) \equiv 0 \bmod p, \\
\frac{\partial f_{\tau}}{\partial x_{i}}(x) \equiv 0 \bmod p, \quad i=1, \ldots, n,
\end{array}\right.
$$

has no solution in $\left(\mathbb{Z}_{p}^{\times}\right)^{n-1} \times \mathbb{Z}_{p}$.

Note that $\tilde{L}_{\tau}$ does not depend on $k$. Thus,

$$
Z_{f}(s, \chi)=\sum_{\substack{\tau \text { face of } \Gamma(f) \\ e_{n} \in \operatorname{rec} \tau}} \tilde{L}_{\tau} S_{\Delta_{\tau}},
$$

with

$$
S_{\Delta_{\tau}}=\sum_{k \in \mathbb{N}^{n} \cap \Delta_{\tau}} p^{-\sigma(k)-m(k) s} .
$$

Suppose moreover that $e_{n} \notin$ rec $\mu$ for each face $\mu$ of $\Gamma(f)$ of maximal pure contribution to the candidate pole $s_{1}$. Then the faces $\tau$ contained in such a $\mu$ do not occur in the sum (7.3). We know that, if $s_{1}$ is a pole of $S_{\Delta_{\tau}}$ for $\tau$ not contained in such a $\mu$, its order is $<\kappa\left(s_{1}\right)$. This is shown in [Hoo02, Proposition 4.9(i)], by choosing 
a partition of $\Delta_{\tau}$ into rational simplicial cones $\Delta_{i}$ such that $<\kappa\left(s_{1}\right)$ generators of $\Delta_{i}$ contribute to $s_{1}$. Now, the assertion about the order follows.

We now prove Assertion [7.4. Fix a face $\tau$ of $\Gamma(f)$ with $e_{n} \in \operatorname{rec} \tau$. Note that because $\sigma \subset\left\{\left(x_{1}, \ldots, x_{n}\right) \in \mathbb{R}^{n} \mid 0 \leq x_{n} \leq 1\right\}$, one has $\operatorname{supp}\left(f_{\tau}\right) \subset H_{0} \cup H_{1}$ and $f_{\tau}=f_{\tau \cap H_{0}}+f_{\tau \cap H_{1}}$.

Case 1. $\operatorname{supp}\left(f_{\tau}\right) \cap H_{1}=\emptyset$. In this case $f_{\tau}=f_{\tau \cap H_{0}}$ is independent of $x_{n}$ and $\tau \cap H_{0}$ is a face of $\Gamma(f)$. Because $f$ is non-degenerate with respect to all the faces of $\Gamma(f)$ contained in $\left\{\left(x_{1}, \ldots, x_{n}\right) \in \mathbb{R}^{n} \mid x_{n}=0\right\}$, the assertion now follows.

Case 2. $\operatorname{supp}\left(f_{\tau}\right) \cap H_{1} \neq \emptyset$. In this case $\left(\partial f_{\tau} / \partial x_{n}\right)=x_{n}^{-1} f_{\tau \cap H_{1}}=x_{n}^{-1} f_{\sigma \cap \tau \cap H_{1}}$ is independent of $x_{n}$ and non-zero on $\left(\mathbb{F}_{p}^{\times}\right)^{n-1}$ because $\sigma \cap \tau \cap H_{1}$ is a face of $\sigma$ (see below) contained in $H_{1}$ and $\sigma$ is unstable over $\mathbb{F}_{p}$ relative to $x_{n}$ and with respect to $f$. Now the assertion follows.

We explain that $\sigma \cap \tau \cap H_{1}$ is a face of $\sigma$. Suppose that $H$ is a supporting hyperplane of $\Gamma(f)$ such that $H \cap \Gamma(f)=\tau$. Then $H$ is also a supporting hyperplane of $\sigma$ such that $H \cap \sigma=\tau \cap \sigma$ because $\sigma \subset \Gamma(f)$. Consequently, $\tau \cap \sigma$ is a face of $\sigma$. Also $\sigma \cap H_{1}$ is a face of $\sigma$ because $\sigma$ is unstable. Due to the fact that the intersection of two faces is again a face, it is now clear that $\sigma \cap \tau \cap H_{1}$ is a face of $\sigma$.

A similar result holds for $Z_{f, 0}(s, \chi)$.

Theorem 7.5. Let $f$ be as in Definition 1.2, and let $p$ be a prime number. Suppose that $f$ is non-degenerate over $\mathbb{F}_{p}$ with respect to all the compact faces of $\Gamma(f)$ contained in $\left\{\left(x_{1}, \ldots, x_{n}\right) \in \mathbb{R}^{n} \mid x_{n}=0\right\}$ and suppose that $f=f_{\sigma}$ where $\sigma \subset \Gamma(f)$ is an unstable convex set over $\mathbb{F}_{p}$ relative to $x_{n}$ and with respect to $f$. (In fact one only has to require condition (ii) in Definition 7.1 for the compact faces of $\sigma$ contained in $\left\{\left(x_{1}, \ldots, x_{n}\right) \in \mathbb{R}^{n} \mid x_{n}=1\right\}$.) Let $s$ be a complex variable with $\operatorname{Re}(s)>0$, and let $\chi$ be a character of $\mathbb{Z}_{p}^{\times}$with conductor $c_{\chi}=1$. Then

$$
Z_{f, 0}(s, \chi)=\sum_{\substack{\tau \text { face of } \Gamma(f) \\\left\{e_{n}\right\}=\operatorname{rec} \tau \cap\left\{e_{1}, \ldots, e_{n}\right\}}} L_{\tau}^{\prime} S_{\Delta_{\tau}}
$$

with

$$
\begin{aligned}
& L_{\tau}^{\prime}=\left\{\begin{array}{cc}
p^{-n}\left((p-1)^{n-1}-p N_{\tau}^{\prime} \frac{p^{s}-1}{p^{s+1}-1}\right) & \text { for } \chi=\chi_{\text {triv }}, \\
p^{-n} \sum_{a \in\left(\mathbb{F}_{p}^{\times}\right)^{n-1} \times\{0\}} \chi\left(f_{\tau}(a)\right) & \text { for } \chi \neq \chi_{\text {triv }},
\end{array}\right. \\
& N_{\tau}^{\prime}=\left\{a \in\left(\mathbb{F}_{p}^{\times}\right)^{n-1} \times\{0\} \mid \bar{f}_{\tau}(a)=0\right\},
\end{aligned}
$$

$S_{\Delta_{\tau}}$ as in Theorem 3.2, $e_{n}=(0,0, \ldots, 1)$ and $\operatorname{rec} \tau$ the set of directions of recession of $\tau$. 
Proof. The proof is almost similar to the proof of Theorem 7.3. We only give a few important steps. It is clear that $Z_{f, 0}(s, \chi)$ equals

$$
\begin{aligned}
& \int_{\left(p \mathbb{Z}_{p}\right)^{n}} \chi(\operatorname{ac} f(x))|f(x)|^{s}|d x| \\
& =\sum_{\left(k_{1}, \ldots, k_{n-1}\right) \in(\mathbb{N} \backslash\{0\})^{n-1}} \int_{\begin{array}{c}
\left(x_{1}, \ldots, x_{n}\right) \in\left(p \mathbb{Z}_{p}\right)^{n} \\
\operatorname{ord}\left(x_{1}, \ldots, x_{n-1}\right)=\left(k_{1}, \ldots, k_{n-1}\right)
\end{array}} \chi(\operatorname{ac} f(x))|f(x)|^{s}|d x| \\
& =\sum_{\substack{\tau \text { face of } \Gamma(f) \\
\left\{e_{n}\right\}=\operatorname{rec} \tau \cap\left\{e_{1}, \ldots, e_{n}\right\}}} \sum_{\left(k_{1}, \ldots, k_{n-1}, 0\right) \in \mathbb{N}^{n} \cap \Delta_{\tau}} \\
& \int_{\substack{\left(x_{1}, \ldots, x_{n}\right) \in\left(p \mathbb{Z}_{p}\right)^{n} \\
\operatorname{ord}\left(x_{1}, \ldots, x_{n-1}\right)=\left(k_{1}, \ldots, k_{n-1}\right)}} \chi(\operatorname{ac} f(x))|f(x)|^{s}|d x| \\
& =\sum_{\substack{\tau \text { face of } \Gamma(f) \\
\left\{e_{n}\right\}=\operatorname{rec} \tau \cap\left\{e_{1}, \ldots, e_{n}\right\}}} \sum_{k \in \mathbb{N}^{n} \cap \Delta_{\tau}} p^{-\sigma(k)-m(k) s} \\
& \int_{\left(\mathbb{Z}_{p}^{\times}\right)^{n-1} \times p \mathbb{Z}_{p}} \chi\left(\operatorname{ac}\left(f_{\tau}\left(u_{1}, \ldots, u_{n-1}, x_{n}\right)+p \tilde{f}_{\tau, k}\left(u_{1}, \ldots, u_{n-1}, x_{n}\right)\right)\right) \\
& \left|f_{\tau}\left(u_{1}, \ldots, u_{n-1}, x_{n}\right)+p \tilde{f}_{\tau, k}\left(u_{1}, \ldots, u_{n-1}, x_{n}\right)\right|^{s}|d u||d x|,
\end{aligned}
$$

where $\tilde{f}_{\tau, k}$ is as in the proof of Theorem [7.3. Denote the last integral by $L_{\tau}^{\prime}$. By a similar argument as in Theorem 7.3 we obtain equality (7.4) of Theorem[7.5 Note that $\tau \cap H_{0}$ is a compact face of $\Gamma(f)$ contained in $\left\{\left(x_{1}, \ldots, x_{n}\right) \in \mathbb{R}^{n} \mid x_{n}=0\right\}$ and $\sigma \cap \tau \cap H_{1}$ is a compact face of $\sigma$ if $\tau$ is a face of $\Gamma(f)$ with $\left\{e_{n}\right\}=\operatorname{rec} \tau \cap$ $\left\{e_{1}, \ldots, e_{n}\right\}$.

We are now able to prove one of the conjectures of Denef and Sargos DS92 Section 6.2] for the $p$-adic case.

Theorem 7.6. Suppose that the prime number $p$, the polynomial $f$ and the character $\chi$ satisfy the conditions of Theorem 3.2. If $\tau_{0}$ is unstable over $\mathbb{F}_{p}$ with respect to $f$, then the following hold:

(i) If $\rho \neq-1$ or $\chi \neq \chi_{\text {triv }}$ and $\rho$ is a pole of $Z_{f}(s, \chi)$, then its order is $<\kappa$.

(ii) If $\rho=-1$ is a pole of $Z_{f}(s)$, then its order is $<\kappa+1$.

Proof. One proves by the same argument as in the proof of Theorem 5.3 that $f_{\tau_{0}}$ is non-degenerate over $\mathbb{F}_{p}$ with respect to all the faces of its Newton polyhedron $\Gamma\left(f_{\tau_{0}}\right)$. Then Theorem 7.6 follows immediately by Theorem 7.3 and Theorem 5.3 Indeed, if $\tau_{0}$ is unstable over $\mathbb{F}_{p}$ relative to $x_{j}$, the unit vector $e_{j}$ does not belong to $\operatorname{rec} \tau$ for faces $\tau \subset \tau_{0}$.

A similar property holds for $Z_{f, 0}(s)$ by Theorem 7.5 and Remark (2) after Theorem 5.3. In this case $f$ has to be non-degenerate with respect to the compact faces of $\Gamma(f)$. Note that $\tau_{0}$ does not have to be compact.

By using Theorem 6.1 instead of Theorem [5.3, we can prove the following result for an arbitrary candidate pole of $Z_{f}(s, \chi)$. 
Theorem 7.7. Suppose that the prime number $p$, the polynomial $f$ and the character $\chi$ satisfy the conditions of Theorem 3.2. Suppose that $s_{1}$ is a candidate pole of $Z_{f}(s, \chi)$, i.e., a number from the set described in Proposition 4.1. Suppose that for every face $\mu$ of $\Gamma(f)$ of maximal pure contribution to the candidate pole $s_{1}$, the face $\mu$ is the only face of $\Gamma\left(f_{\mu}\right)$ of maximal pure contribution to the candidate pole $s_{1}$, and $\mu$ is unstable over $\mathbb{F}_{p}$ with respect to $f$. Then the following hold:

(i) If $p^{s_{1}+1} \neq 1$ or $\chi \neq \chi_{\text {triv }}$ and $s_{1}$ is a pole of $Z_{f}(s, \chi)$, then its order is $<\kappa\left(s_{1}\right)$.

(ii) If $p^{s_{1}+1}=1$ and $s_{1}$ is a pole of $Z_{f}(s)$, then its order is $<\kappa\left(s_{1}\right)+1$.

Remark. The proof of Theorem 6.1 shows that the condition " $\mu$ is the only face of $\Gamma(f)$ of maximal pure contribution to the candidate pole $s_{1}$ " in Theorem 7.7 can be replaced by a weaker condition. Examples show that even this condition might be omitted. (For example, take $f(x, y, z)=x^{7} y^{3} z+x^{4} y^{8}+x^{8} y^{4}+z^{7}$ as in the example from Remark (1) after Theorem 6.1 Then the condition on $\mu$ is not satisfied for the candidate pole $-1 / 3$. Nevertheless, we calculated with the program Polygusa [HL00] that $-1 / 3$ is not an actual pole of Igusa's local zeta function $Z_{f}(s)$, e.g., for $p=3, p=5$ and $p=31$.) We would like to search for a proof or a counterexample.

A similar property holds for $Z_{f, 0}(s)$. In this case $f$ has to be non-degenerate with respect to the compact faces of $\Gamma(f)$.

Remark. In the very special case that there is only one facet of pure contribution to $s_{1}$ that is moreover an unstable simplex, J. Denef obtained the following related result.

Theorem 7.8. Let $f$ be a polynomial over $\mathbb{Z}_{p}$ in $n$ variables. Suppose that $f$ is non-degenerate over $\mathbb{Q}_{p}$ with respect to the compact faces of its Newton polyhedron. Suppose that $s_{1} \neq 1$ is a real candidate pole of $Z_{f}(s)$ such that there is only one facet $\mu$ of pure contribution to $s_{1}$. Suppose that $\mu$ is a simplex with $n-1$ vertices contained in a coordinate hyperplane and one vertex at distance 1 from that coordinate hyperplane. Then there is no pole of

$$
\int_{p^{k} \mathbb{Z}_{p}^{n}}|f(x)|^{s}|d x|
$$

with real part equal to $s_{1}$ for $k \gg 0$.

\section{EXAMPLES}

We use Theorem 3.2 and Theorem 7.3 to calculate Igusa's local zeta function in the following examples. To find the cones associated to the proper faces of the Newton polyhedron, we use Proposition 2.7 To calculate the $S_{\Delta_{\tau}}$ we divide these cones into rational simplicial cones. To find the number of the solutions of the set $\mathbb{Z}^{n} \cap\left\{\sum \lambda_{i} a_{i} \mid 0 \leq \lambda_{i}<1\right\}$, the remark after Definition 2.10 can be useful. More examples can be found in [HL00] and [DH01]. The computer program "Polygusa" HL00 to calculate $Z_{f}(s)$ for a concrete polynomial $f$ is written in Maple and is based on the formula from Theorem 3.2 


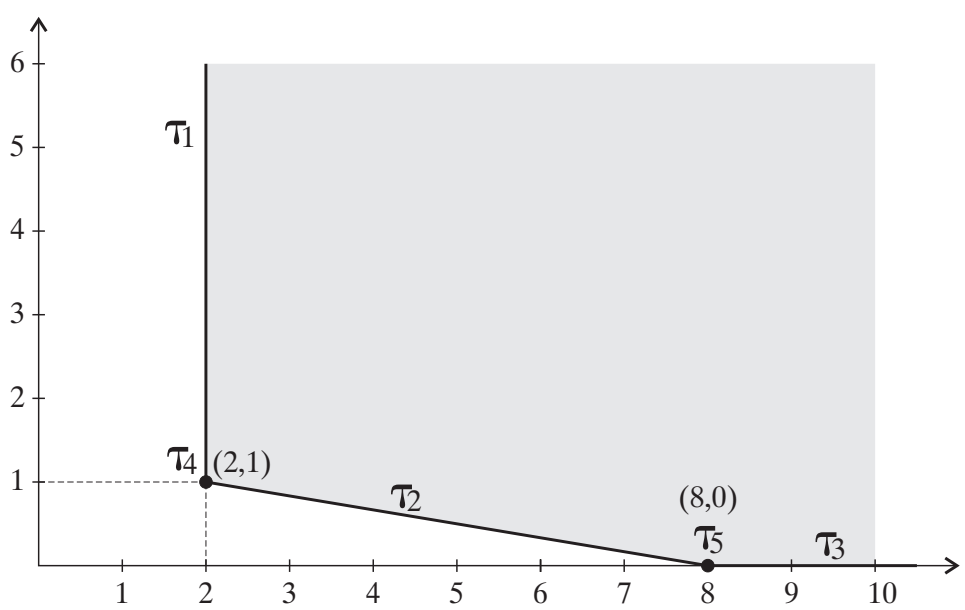

FiguRE 8.1. Newton polyhedron of $x^{2} y+x^{8}$

Example 8.1. Let $f_{1}(x, y)=x^{2} y+x^{8}$. It is easy to verify that the Newton polyhedron $\Gamma\left(f_{1}\right)$ of $f_{1}$ is defined by the system of linear inequalities

$$
\left\{\begin{array}{l}
x \geq 2 \\
y \geq 0 \\
x+6 y \geq 8
\end{array}\right.
$$

A picture of the Newton polyhedron of $f_{1}$ and its proper faces $\tau_{1}, \ldots, \tau_{5}$ is given in Figure 8.1 If $p$ is prime, then it is easy to verify that $f_{1}$ is non-degenerate over $\mathbb{F}_{p}$ with respect to all the faces of its Newton polyhedron. So, we can use the formula for $Z_{f_{1}}(s)$ from Theorem 3.2 More information about the cones associated to the proper faces is contained in Table 8.1. The cone $\Delta_{\tau_{4}}$ is rational simplicial, but the multiplicity of $(1,0),(1,6)$ is 6 and

$$
\begin{aligned}
\mathbb{Z}^{2} & \cap\left\{\lambda_{1}(1,0)+\lambda_{2}(1,6) \mid 0 \leq \lambda_{i}<1, i=1,2\right\} \\
& =\{(0,0),(1,1),(1,2),(1,3),(1,4),(1,5)\} .
\end{aligned}
$$

\begin{tabular}{ccc}
\hline Proper face $\tau$ & Generators for $\Delta_{\tau}$ & $S_{\Delta_{\tau}}$ \\
\hline$\tau_{1}$ & $(1,0)$ & $\frac{1}{p^{1+2 s}-1}$ \\
$\tau_{2}$ & $(1,6)$ & $\frac{1}{p^{7+8 s}-1}$ \\
$\tau_{3}$ & $(0,1)$ & $\frac{1}{p-1}$ \\
$\tau_{4}$ & $(1,0),(1,6)$ & $\frac{1+p^{2+3 s}+p^{3+4 s}+p^{4+5 s}+p^{5+6 s}+p^{6+7 s}}{\left(p^{1+2 s}-1\right)\left(p^{7+8 s}-1\right)}$ \\
$\tau_{5}$ & $(0,1),(1,6)$ & $\frac{1}{(p-1)\left(p^{7+8 s}-1\right)}$ \\
\hline
\end{tabular}

TABLE 8.1. The cones associated to the faces of $\Gamma\left(x^{2} y+x^{8}\right)$

The other cones $\Delta_{\tau}$ are all simple cones. It is easy to see that $N_{\tau_{2}}=N_{\Gamma\left(f_{1}\right)}=$ $\left\{(x, y) \in\left(\mathbb{F}_{p}^{\times}\right)^{2} \mid x^{2} y+x^{8}\right\}=p-1$ and $N_{\tau_{1}}=N_{\tau_{3}}=N_{\tau_{4}}=N_{\tau_{5}}=0$. According to the formula for $Z_{f_{1}}(s)$ in Theorem 3.2 the real candidate poles of $Z_{f_{1}}(s)$ are $-1,-1 / 2$ and $-7 / 8$, all with expected order 1 . However, $\Gamma_{\mathrm{gl}}\left(f_{1}\right)$ is unstable over 
$\mathbb{F}_{p}$ relative to $y$ and with respect to $f_{1}$. So, if we use the formula for $Z_{f_{1}}(s)$ from Theorem 7.3, we get

$$
Z_{f_{1}}(s)=\tilde{L}_{\Gamma\left(f_{1}\right)}+\tilde{L}_{\tau_{1}} S_{\Delta_{\tau_{1}}}
$$

According to this formula, it is not surprising that $-7 / 8$ is not an actual pole of $Z_{f_{1}}(s)$. Indeed,

$$
Z_{f_{1}}(s)=\frac{p^{3 s}(p-1)^{2}}{\left(p^{1+2 s}-1\right)\left(p^{1+s}-1\right)} .
$$

Let $f_{2}(x, y)=x^{2} y+x^{8}+x^{10}$ and $f_{3}(x, y)=x^{2} y+x^{8}+x^{10} y^{9}$. If $p$ is prime and $p \neq 2$, then it is easy to verify that $f_{2}$ is non-degenerate over $\mathbb{F}_{p}$ with respect to all the faces of its Newton polyhedron. If $p$ is prime and $p \gg 0$, then $f_{3}$ is non-degenerate over $\mathbb{F}_{p}$ with respect to all the faces of its Newton polyhedron. So, we can use the formulas for $Z_{f_{2}}(s)$ and $Z_{f_{3}}(s)$ from Theorem 3.2 for those primes. The Newton polyhedra of $f_{1}, f_{2}$ and $f_{3}$ are all equal, and so also are the $S_{\Delta_{\tau}}$. Again according to the formulas for $Z_{f_{2}}(s)$ and $Z_{f_{3}}(s)$ from Theorem 3.2 the number $-7 / 8$ is a real candidate pole of $Z_{f_{2}}(s)$ and $Z_{f_{3}}(s)$ with expected order 1 . But by the same argument as for $f_{1}$, it is not surprising that the real number $-7 / 8$ is not an actual pole of $Z_{f_{2}}(s)$ because $\Gamma_{\mathrm{gl}}\left(f_{2}\right)$ is unstable over $\mathbb{F}_{p}$ relative to $y$ and with respect to $f_{2}$. Indeed,

$$
Z_{f_{2}}(s)=\frac{p^{3 s}(p-1)^{2}}{\left(p^{1+2 s}-1\right)\left(p^{1+s}-1\right)} .
$$

The set $\Gamma_{\mathrm{gl}}\left(f_{3}\right)$ is not unstable, but

$$
\lim _{s \rightarrow-7 / 8}\left(p^{s+7 / 8}-1\right) Z_{f_{3}}(s)=\lim _{s \rightarrow-7 / 8}\left(p^{s+7 / 8}-1\right) Z_{f_{1}}(s),
$$

because $L_{\tau_{2}}\left(f_{3}\right)=L_{\tau_{2}}\left(f_{1}\right), L_{\tau_{4}}\left(f_{3}\right)=L_{\tau_{4}}\left(f_{1}\right), L_{\tau_{5}}\left(f_{3}\right)=L_{\tau_{5}}\left(f_{1}\right)$. One may also obtain (8.1) by applying Theorem 6.1 to $f_{3}$ and $f_{1}$. So, $-7 / 8$ is not an actual pole of $Z_{f_{3}}(s)$ either.

Remark. The zero-sets of the polynomials $f_{1}, f_{2}$ and $f_{3}$ are normal crossing divisors. By the work of Igusa Igu74, one can also obtain that $-1 / 2$ and -1 are the only possible poles. One can also easily obtain the results above by the formula of Denef Den87.

Example 8.2. Let $f_{4}(x, y, z)=x y+x z^{2}+y z^{2}$. The Newton polyhedron $\Gamma\left(f_{4}\right)$ of $f_{4}$ is defined by the system of linear inequalities

$$
\left\{\begin{array}{l}
x \geq 0 \\
y \geq 0 \\
z \geq 0 \\
2 x+2 y+z \geq 4 \\
2 y+z \geq 2 \\
2 x+z \geq 2 \\
x+y \geq 1
\end{array}\right.
$$

We denote the facets with supporting hyperplanes given by the corresponding equalities in the inequalities above by $\tau_{1}, \ldots, \tau_{7}$. A picture of the Newton polyhedron of $f_{4}$ is given in Figure 8.2 ,

There are 19 proper faces. One easily verifies that $f_{4}$ is non-degenerate over $\mathbb{F}_{p}$ with respect to all the faces of its Newton polyhedron, for every prime $p \neq 2$. So, we can use the formula for $Z_{f_{4}}(s)$ from Theorem 3.2 for those primes. According to this formula, the candidate real poles of $Z_{f_{4}}(s)$ are $-1,-5 / 4,-3 / 2$ and -2 , 


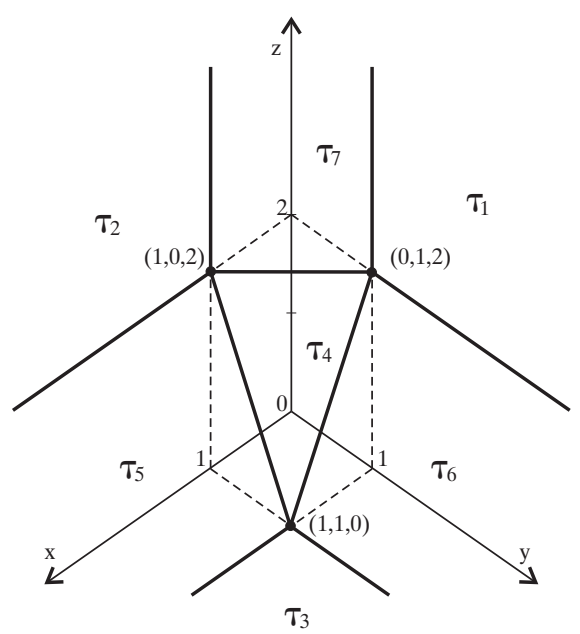

FiguRE 8.2. Newton polyhedron of $x y+x z^{2}+y z^{2}$

all with expected order 1 . The set of faces of $\Gamma\left(f_{4}\right)$ of maximal pure contribution to the candidate poles $-5 / 4,-3 / 2,-2$ are $\left\{\tau_{4}\right\},\left\{\tau_{5}, \tau_{6}\right\},\left\{\tau_{7}\right\}$, respectively (see Definition 4.2, Definition 4.6 and Proposition 4.7). Note that this example shows that two faces of $\Gamma\left(f_{4}\right)$ of maximal pure contribution to the same candidate pole do not have to be disjoint. However, $-3 / 2$ and -2 are not actual poles of $Z_{f_{4}}(s)$. Indeed, by using the formula for $Z_{f_{4}}(s)$ from Theorem [3.2 (see [HL00]), we get

$$
Z_{f_{4}}(s)=\frac{p^{2 s}(p-1)\left(p^{5+3 s}+p^{2+s}-p^{2+2 s}-p^{1+s}+p-1\right)}{\left(p^{1+s}-1\right)\left(p^{5+4 s}-1\right)} .
$$

Theorem 7.7 explains why this happens. For example, $-3 / 2$ is not an actual pole because the faces $\tau_{5}, \tau_{6}$ of $\Gamma\left(f_{4}\right)$ of maximal pure contribution to the candidate pole $-3 / 2$ are unstable and in addition the following property is satisfied. The face $\tau_{5}$ (resp. $\left.\tau_{6}\right)$ is the only face of $\Gamma\left(\left(f_{4}\right)_{\tau_{5}}\right)$ (resp. $\Gamma\left(\left(f_{4}\right)_{\tau_{6}}\right)$ ) of maximal pure contribution to $-3 / 2$. A similar argument holds for -2 .

\section{REFERENCES}

[Den84] J. Denef, The rationality of the Poincaré series associated to the p-adic points on a variety, Invent. Math. 77 (1984), 1-23. MR 86c:11043

[Den87] _ On the degree of Igusa's local zeta function, Amer. J. Math. 109 (1987), 9911008. MR 89d:11108

[Den91] — Report on Igusa's local zeta function, Astérisque 201-203 (1991), 359-386, Séminaire Bourbaki 1990/1991. MR 93g:11119

[Den95] — Poles of p-adic complex powers and Newton polyhedra, Nieuw Arch. Wisk. 13 (1995), no. 3, 289-295. MR 96m:11106

[DH01] J. Denef and K. Hoornaert, Newton polyhedra and Igusa's local zeta function, J. Number Theory 89 (2001), 31-64. MR 2002g: 11170

[DLS97] J. Denef, A. Laeremans, and P. Sargos, On the largest nontrivial pole of the distribution $|f|^{s}$, RIMS Kokyuroku 999 (1997), 1-9. MR 99g:11101

[DS92] J. Denef and P. Sargos, Polyèdre de Newton et distribution $f_{+}^{s}$. II, Math. Ann. 293 (1992), 193-211. MR 93f:32037

[HL00] K. Hoornaert and D. Loots, Polygusa: a computer program for Igusa's local zeta function, http://www.wis.kuleuven.ac.be/wis/algebra/kathleen.htm, 2000. 
[Hoo02] K. Hoornaert, Newton polyhedra and the poles of Igusa's local zeta function, Bull. Belg. Math. Soc. - Simon Stevin 9 (2002), 589-606.

[Igu74] J.-I. Igusa, Complex powers and asympotic expansions I, J. Reine Angew. Math 268/269 (1974), 110-130; II, ibid, 278/279, 307-321,1975. MR 50:254 MR 53:8018

[Igu78] Lectures on forms of higher degree, Tata Inst. Fund. Res. Lectures on Math. and Phys., vol. 59, Springer-Verlag, Heidelberg-New York-Berlin, 1978. MR 80m:10020

[LM85] B. Lichtin and D. Meuser, Poles of a local zeta function and Newton polygons, Compositio Math. 55 (1985), 313-332. MR 87a:11120

[PS00] D.H. Phong and J.A. Sturm, Algebraic estimates, stability of local zeta functions, and uniform estimates for distribution functions, Ann. of Math. 152 (2000), 277-329. MR 2002f:11180

[PSS99] D.H. Phong, E.M. Stein, and J.A. Sturm, On the growth and stability of real analytic functions, Amer. J. Math. 121 (1999), 519-554. MR 2002a:58025

[Roc70] R.T. Rockafellar, Convex analysis, Princeton University Press, Princeton, N.J., 1970. MR 43:445

[Zn99] W.A. Zúñiga-Galindo, Local zeta functions and Newton polyhedra, preprint, 1999.

Department of Mathematics, Catholic University Leuven, Celestijnenlaan 200B, 3001 Leuven, Belgium 